Review

\title{
Quantum Cosmology vs. Observational Cosmology (A Simple, Curious and Advanced Approach)
}

\author{
U. V. S. Seshavatharam ${ }^{1, *}$ and S. Lakshminarayana ${ }^{2}$ \\ 1 Honorary faculty, I-SERVE, Alakapuri, Hyderabad-35, Telangana, India \\ 2 Dept. of Nuclear Physics, Andhra University, Visakhapatnam-03, AP, India; Insrirama@gmail.com \\ * Correspondence: seshavatharam.uvs@gmail.com
}

\begin{abstract}
With reference to Planck scale Hubble parameter, super luminal expansion speeds, super luminal rotation speeds, Mach's principle and Holographic principle, we review the current cosmological observations with eight simple assumptions. By understanding Yuri N. Obukhov and V.A. Korotky proposed cosmic rotational effects of polarization of radiation due to massive bodies, to some extent cosmic rotation can be deep-rooted in an observational approach and the ratio of current angular velocity and Hubble parameter can be estimated. It is possible to show that, at $H_{0}=70 \mathrm{~km} / \mathrm{sec} / \mathrm{Mpc}$, current cosmic temperature, age, radius, mass, mass density are $2.721 \mathrm{~K}, 4.41 \times 10^{17} \mathrm{sec}, 90$ billion light years, $1.14654 \times 10^{54} \mathrm{~kg}, 0.0482$ times the current critical density respectively. Clearly speaking, current universe seems to constitute 267 Hubble spheres. Important point to be noted is that, current rotational kinetic energy is 0.6667 times the current critical energy. Based on the estimated current mass density and current rotational kinetic energy, current cosmic dark matter density can be shown to be 0.2851 times the current critical density. These numerical coincidences cast serious doubt on the on the real existence of currently believed 'dark energy'. Initial and current expansion speeds are $3 \times 10^{8} \mathrm{~m} / \mathrm{sec}$ and $3.56 \times 10^{9} \mathrm{~m} / \mathrm{sec}$ respectively. With increasing cosmic age and increasing cosmic expansion speed, current universe is expanding with a speed of $11.885 \mathrm{c}$. By knowing the time to time future cosmic temperatures, future Hubble parameters and corresponding future cosmic expansion speeds can be estimated and thus future expansion speed can be understood. Starting from 'speed of light', our model assumes a continuous increase in expansion speed and attains a current radius of 90 billion light years (without inflationary concepts) and casts a serious doubt on the actuality of currently believed 'inflation'.
\end{abstract}

Keywords: big bang; planck scale Hubble parameter; quantum cosmology; Mach's principle; holographic principle; observational cosmology; super luminal expansion speed; super luminal rotation speed; dark energy; cosmic rotational kinetic energy; cosmic microwave background radiation; cosmic age and scale factor; polarization rotation effect

\section{Introduction}

Photons and black holes can be considered as the best candidates of quantum gravitational objects. It is true that, without the existence of universe, there is no independent existence to any photon or any black hole. Now the fundamental question to be answered is: Is our universe a quantum gravitational object or something else? Physicists expressed several opinions with many possible solutions [1-5] and references therein. We could also express 
different unified views in this direction [6-8] and readers are strongly encouraged to go through.

In an optimistic approach, some of the modern cosmologists believe that, during cosmic evolution, Planck scale quantum gravitational interactions might have an observable effect on the current observable cosmological phenomena. Clearly speaking, with respect to 'Quantum gravity' and Planck scale early universal laboratory, current universe can be considered as a low energy scale laboratory. If one is willing to consider the current observable universe as a low energy scale laboratory, currently believed cosmic microwave back ground temperature can be considered as the low energy quantum gravitational effect. At any time in the past, i.e as the operating energy scale was assumed to be increasing; past high cosmic back ground temperature can be considered as the high energy quantum gravitational effect. Thinking in this way, starting from the Planck scale, 'quantum cosmology' can be considered as 'scale independent' and the universe can be considered as the best quantum gravitational object. In this context, we have chosen the following two quantitative relations.

1) We define the Planck scale Hubble parameter, $H_{p l} \cong \sqrt{c^{5} / G \hbar} \cong 1.8549215 \times 10^{43} \sec ^{-1}$ and apply it to cosmological data fitting in the form of $\left[1+\ln \left(\frac{H_{p l}}{H_{t}}\right)\right]^{n}$ where $H_{t}$ is the running Hubble parameter and $n$ is a suitable power index.

2) According to $G$ 't Hooft, the combination of quantum mechanics and gravity requires the three dimensional world to be an image of data that can be stored on a two dimensional projection much like a holographic image $[9,10]$. The 'holographic principle' is a property of string theory and a supposed property of quantum gravity that states that the description of a volume of space can be thought of as encoded on a lower-dimensional boundary. Based on this concept, for the four dimensional spacetime universe, its three dimensional increasing volume can be set by Mach's principle, $\frac{G M_{t}}{R_{t} c^{2}} \cong 1$. Clearly speaking, information of the evolving universe, can be extracted from $R_{t} \cong \frac{G M_{t}}{c^{2}}$. With this proposal, at any stage of cosmic evolution, a closed and massive universe can be defined. One can find interesting technical discussion on this assumption by D.W.Sciama, R.H. Dicke, C. Brans and G. J. Whitrow [11-18].

Based on these quantitative relations, we re-view the phenomena of 'inflation' [19-21] 'acceleration' and 'dark energy' [22-25] with increasing super luminal expansion speeds and increasing super luminal rotational speeds. In this revised version, we proposed methods for understanding cosmic age corresponding to a redshift of 1100 and temperature of $3000 \mathrm{~K}$. In this context, readers are strongly encouraged to see our short communication [26] and its elaborated early version [27].

We arranged our revised version in the following way. In section-2, we proposed our eight assumptions connected with big bang and Planck scale. In section-3 we proposed many 
possible applications pertaining to observational cosmology. In section-4, we presented the views of Yuri N. Obukhov and V.A. Korotky on 'polarization rotation effect'. In section-5, we discussed on 'horizon problem'. In section- 6 we presented the views of Steinhardt PJ on 'inflation.' In section-7, we presented the views of Martin Bozowald on quantum cosmology. Section-8 constitutes a lengthy discussion on the scope and applicability of proposed assumptions. In section-9 we presented our concluding remarks.

Subject of cosmology is quite interesting, very complicated and quite controversial.

A) In June 2015, three professors, Jeppe Trost Nielsen, Alberto Guffanti and Subir Sarkar of Niels Bohr International Academy and Rudolf Peierls Centre for Theoretical Physics, using the JLA catalogue of 740 SN Ia processed by the SALT2 method, come to a conclusion that $[28,29]$, evidence for the currently believed cosmic acceleration is only marginal and current universe seems to expand at a constant rate. In their words: "The 'standard' model of cosmology is founded on the basis that the expansion rate of the universe is accelerating at present - as was inferred originally from the Hubble diagram of Type Ia supernovae. There exists now a much bigger database of supernovae so we can perform rigorous statistical tests to check whether these 'standardisable candles' indeed indicate cosmic acceleration. Taking account of the empirical procedure by which corrections are made to their absolute magnitudes to allow for the varying shape of the light curve and extinction by dust, we find, rather surprisingly, that the data are still quite consistent with a constant rate of expansion."

B) Over the last sixty plus years, numerous rotating and expanding general relativity-compatible cosmological models have been developed [30-70]. An excellent review of this history is provided in Yuri Obukhov's paper [30]. According to Yuri N. Obukhov and V.A. Korotky: the cosmic rotation affects a polarization of radiation which propagates in the curved spacetime and this produces a new observable effect which has been reported in the literature by Birch [35,36] and Nodland and Ralston [37,38]. This type of observation can be considered as one best possible observational evidence for cosmic rotation. According to their analysis, current angular velocity seems to be, $\omega_{0} \cong(1.8 \pm 0.8) H_{0}$.

In this context, with reference to our proposed assumptions $3 \mathrm{~d}$ and $3 \mathrm{f}$, at $H_{0}=70 \mathrm{~km} / \mathrm{sec} / \mathrm{Mpc}$, current cosmic expansion speed is $11.885 \mathrm{c}$ and temperature is $2.721 \mathrm{~K}$. For a moment, let us guess that, in coming future, (current) Hubble parameter decreases by a factor of 10 and reaches to $H_{0}=7 \mathrm{~km} / \mathrm{sec} / \mathrm{Mpc}$,. Then with our assumptions $3 \mathrm{~d}$ and $3 \mathrm{f}$, corresponding cosmic expansion speed and temperature can be estimated to be $11.98 c$ and $0.854 \mathrm{~K}$ respectively. Clearly speaking, expansion speed increases from $11.885 \mathrm{c}$ to $11.98 \mathrm{c}$ and practically seems to represent a 'constant rate of expansion'. See the following figure -1 for the increasing cosmic speed. 
Figure 1:-Increasing cosmic expansion speed

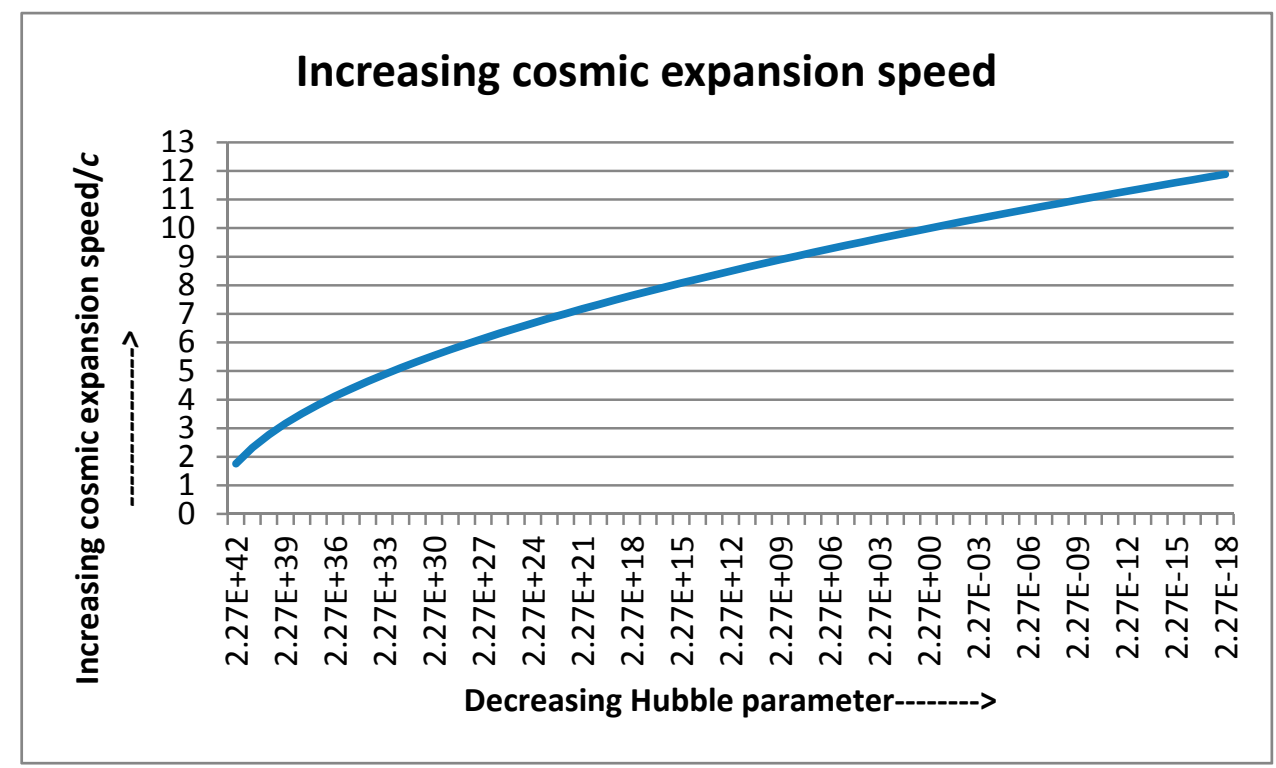

\section{Workable assumptions connected with Planck scale}

With the following eight simple and logical assumptions, most of the currently believed cosmological observations can be reviewed and refined at fundamental level. In developing any novel model, one should see the possibility of minimizing the number of assumptions. In this context, we would like say that, our proposed assumptions are very clear and very simple.We are trying our level best in understanding and refining their individual roles and collective role. We are confident to say that, with further research and analysis, there is a possibility of reducing the number of assumptions to four or five. For the time being we request the readers to go through the rest of the paper and please evaluate its novellty with reference to: 1) Implementing Planck scale; 2) Implemeting Mach's principle; 3) Developing a model of quantum cosmology; 4) Current cosmological data fitting; 5) Extrapolation to past and future; 6) Compatibility with hot big bang model; 7) Comtability with dark matter; 8) Simplicity; 9) Ability for extension or modication;

1) With reference to big bang and Planck scale, Hubble parameter associated with Planck scale can be defined as $H_{p l} \cong \sqrt{c^{5} / G \hbar} \cong 1.8549215 \times 10^{43} \mathrm{sec}^{-1}$

2) Speed of light can be considered as the initial cosmic expansion speed.

3) At any stage of cosmic evolution, from and about the point of big bang,

a) $\left[1+\ln \left(H_{t} / H_{0}\right)^{2}\right]\left(\frac{1}{H_{t}}\right)$ can be considered as the cosmic age. It may be noted that, for the current case, $H_{t} \cong H_{0}$ and $\left[1+\ln \left(H_{t} / H_{0}\right)^{2}\right]\left(\frac{1}{H_{t}}\right) \cong\left(\frac{1}{H_{0}}\right)$.

b) $\frac{G M_{t}}{R_{t} c^{2}} \cong 1$ where $M_{t}$ is the cosmic mass and $R_{t}$ is the cosmic radius or distance travelled. 
c) Magnitude of cosmic angular velocity $\omega_{t}$ is equal to $H_{t}$.

d) $V_{t} \cong \sqrt{1+\ln \left(\frac{H_{p l}}{H_{t}}\right)} \times c$ can be considered as the cosmic expansion speed.

e) Distance travelled, $S_{t} \cong\left(\frac{V_{t}+c}{2}\right) t$ can be considered as the cosmic radius $R_{t}$.

f) Ratio of critical energy density and thermal energy density is equal to $\left[1+\ln \left(\frac{H_{p l}}{H_{t}}\right)\right]^{2}$.

Note-1: Based on the Mach's principle and GTR, it is possible to guess that, increasing matter content increases the radius of curvature and there exists no room for causal disconnection. Quantitatively this can be understood with assumption 3b. With this idea, 'horizon problem' can be resolved. See section-5.

Note-2: Mainstream cosmologists believe that the superluminal expansion period of the universe (called "cosmic inflation") ended by $10^{-32}$ seconds (a tiny fraction of a second) after the hot big bang [19-21]. Since that time, they believe, expansion initially decelerated (from gravity) and then, after about 6 billion years, began very slowly to accelerate (from dark energy). Many cosmologists proposed different starting mechanisms for initiating and fine tuning the believed 'inflation'. In this context, we would like to stress the fact that, starting from 'speed of light', our model assumes a continuous increase in expansion speed attains a current radius of 90 billion light years which is just twice of the modern estimate! Clearly speaking, considering assumptions $2,3 \mathrm{~b}, 3 \mathrm{c}$ and $3 \mathrm{~d}$, currently believed cosmic inflation, acceleration, dark energy, cosmic homogeneity and cosmic horizon problems can be reviewed and re-addressed in a very simplified approach. In addition, problems connected with 'fine tuning' of beginning of 'inflation' can be ignored. See section- 6

Steps involved in estimating the cosmic physical parameters are:

1) With reference to cosmic temperature, corresponding Hubble parameter can be estimated.

2) By knowing the Hubble parameter, corresponding cosmic age and cosmic expansion speed can be estimated.

3) By knowing cosmic age and expansion speed, corresponding cosmic radius can be estimated.

4) By knowing the cosmic radius, corresponding cosmic mass can be estimated.

5) By knowing the cosmic mass, corresponding mass density and rotational kinetic energy can be estimated.

By knowing the cosmic mass density and rotational kinetic energy density, corresponding dark matter density can be estimated. 


\section{Various applications of $\left[1+\ln \left(H_{p l} / H_{t}\right)\right]$ in cosmology}

\section{Application-1: Relation between cosmic thermal energy density and critical energy density}

Let us assume that, during cosmic evolution, at any time, thermal energy density is proportional to the critical energy density.

$$
a T_{t}^{4} \propto \frac{3 H_{t}^{2} c^{2}}{8 \pi G}
$$

With reference to the Planck scale and by considering the proportionality factor as $\left[1+\ln \left(\frac{H_{p l}}{H_{t}}\right)\right]^{-2}$, it is possible to define that,

$$
\begin{aligned}
& a T_{t}^{4} \cong\left[1+\ln \left(\frac{H_{p l}}{H_{t}}\right)\right]^{-2}\left(\frac{3 H_{t}^{2} c^{2}}{8 \pi G}\right) \cong\left(\frac{c}{V_{t}}\right)^{4}\left(\frac{3 H_{t}^{2} c^{2}}{8 \pi G}\right) \\
& \rightarrow T_{t} \cong\left[1+\ln \left(\frac{H_{p l}}{H_{t}}\right)\right]^{-1 / 2}\left(\frac{3 H_{t}^{2} c^{2}}{8 \pi G a}\right)^{\frac{1}{4}} \cong\left(\frac{c}{V_{t}}\right)\left(\frac{3 H_{t}^{2} c^{2}}{8 \pi G a}\right)^{\frac{1}{4}}
\end{aligned}
$$

where $V_{t}$ is the cosmic expansion speed and $c$ is the initial cosmic expansion speed.

$$
\sqrt{\frac{3 H_{t}^{2} c^{2}}{8 \pi G a T_{t}^{4}}} \cong\left[1+\ln \left(\frac{H_{p l}}{H_{t}}\right)\right] \cong\left(\frac{V_{t}}{c}\right)^{2}
$$

For the current case,

$$
\begin{aligned}
& a T_{0}^{4} \cong\left[1+\ln \left(\frac{H_{p l}}{H_{0}}\right)\right]^{-2}\left(\frac{3 H_{0}^{2} c^{2}}{8 \pi G}\right) \cong\left(\frac{c}{V_{0}}\right)^{4}\left(\frac{3 H_{0}^{2} c^{2}}{8 \pi G}\right) \\
& \rightarrow T_{0} \cong\left[1+\ln \left(\frac{H_{p l}}{H_{0}}\right)\right]^{-1 / 2}\left(\frac{3 H_{0}^{2} c^{2}}{8 \pi G a}\right)^{\frac{1}{4}} \cong\left(\frac{c}{V_{0}}\right)\left(\frac{3 H_{0}^{2} c^{2}}{8 \pi G a}\right)^{\frac{1}{4}}
\end{aligned}
$$

where $V_{0}$ is the current cosmic expansion speed.

$$
\sqrt{\frac{3 H_{0}^{2} c^{2}}{8 \pi G a T_{0}^{4}}} \cong\left[1+\ln \left(\frac{H_{p l}}{H_{0}}\right)\right] \cong\left(\frac{V_{0}}{c}\right)^{2}
$$

With trial-error, it is noticed that, at

$H_{0} \cong 70.0 \mathrm{~km} / \mathrm{sec} / \mathrm{Mpc} \cong 2.268529 \times 10^{-18} \mathrm{sec}^{-1},\left(1 \mathrm{Mpc}=3.085677581 \times 10^{22} \mathrm{~m}\right)$

$$
\begin{aligned}
& \text { Obtauned } T_{0} \cong 2.72075 \mathrm{~K} \text { and } \\
& \sqrt{1+\ln \left(\frac{H_{p l}}{H_{0}}\right)} \cong\left(\frac{V_{0}}{c}\right) \cong 11.8851 \\
& \rightarrow V_{0} \cong 3.563073 \times 10^{9} \mathrm{~m} \cdot \mathrm{sec}^{-1}
\end{aligned}
$$


As per the 2015 Planck data [23], the current value of CMBR temperature is:

$$
\left.\begin{array}{l}
\text { Planck TT + lowP + BAO: }(2.722 \pm 0.027) \mathrm{K} \\
\text { Planck TT; TE; EE + low P + BAO: }(2.718 \pm 0.021) \mathrm{K}
\end{array}\right\}
$$

This fitted value of $H_{0} \cong 70.0 \mathrm{~km} / \mathrm{sec} / \mathrm{Mpc}$ can be compared with the very recent reference [23]. As per reference [24], best value of $H_{0} \cong(73.24 \pm 1.74) \mathrm{km} / \mathrm{sec} / \mathrm{Mpc}$. Clearly speaking, $\left\{(67.7 \pm 0.66)>H_{0}<(73.24 \pm 1.74)\right\} \mathrm{km} / \mathrm{sec} / \mathrm{Mpc}$.

As per the 2015 Planck data [23], the current value of the Hubble parameter is reported to be:

$$
\left.\begin{array}{l}
\text { Planck TT+low P: }(67.31 \pm 0.96) \mathrm{km} / \mathrm{sec} / \mathrm{Mpc} \\
\text { Planck TE+low P: }(67.73 \pm 0.92) \mathrm{km} / \mathrm{sec} / \mathrm{Mpc} \\
\text { Planck TT,TE,EE+low P: }(67.7 \pm 0.66) \mathrm{km} / \mathrm{sec} / \mathrm{Mpc}
\end{array}\right\}
$$

Clearly speaking, our fit of $H_{0}$ seems to lie in between the values recommended in reference [23] and reference [24] respectively. See section 7 for further discussion on relation (2).

Note: As the universe is always assumed to be expanding at 'increasing super luminal speeds', there is no scope for 'temperature isotropy' and cosmic temperature will decrease continuously. Since the current observable universe is very large and as the observer is not in a position to reach all parts of the current universe, one may be forced to arrive at a misconception of 'CMBR isotropy'.

It may be noted that, in a quantum gravitational approach, relation (2) can be expressed in the following general form.

$$
\begin{aligned}
& T_{t} \cong\left[0.46148\left(\frac{\sqrt{1+\sqrt{y}}}{\sqrt{y}}\right)\right] \frac{\hbar c^{3}}{k_{B} G \sqrt{M_{t} M_{p l}}} \\
& \text { where, } y \cong\left[1+\ln \left(\frac{H_{p l}}{H_{t}}\right)\right], M_{t} \cong \frac{c^{3}}{2 G H_{t}}\left[1+\sqrt{1+\ln \left(\frac{H_{p l}}{H_{t}}\right)}\right] \text { and } M_{p l} \cong \sqrt{\frac{\hbar c}{G}}
\end{aligned}
$$

Here in this relation, we try to highlight the expression, $\left[\frac{\hbar c^{3}}{k_{B} G \sqrt{M_{t} M_{p l}}}\right]$. Qualitatively this expression is similar to Hawking's black hole temperature formula [71] and needs further study. In our earlier publications, \{author Seshavatharam U.V.S [72,73] and along with S. Lakshminarayana and E. Terry Tatum [74-78]\}, we proposed that,

$$
T_{t} \cong \frac{\hbar c^{3}}{8 \pi k_{B} G \sqrt{M_{t} M_{p l}}}
$$


where $M_{t}$ is the mass of universe and is equal to $\left(\frac{c^{3}}{2 G H_{t}}\right)$.

\section{Application-2: To estimate the current cosmic age}

From the beginning of Planck scale which is assumed to be associated with big bang, cosmic age can be estimated as follows:

$$
\begin{aligned}
& t_{0} \cong\left[1+\ln \left(H_{0} / H_{0}\right)^{2}\right]\left(\frac{1}{H_{0}}\right) \cong\left(\frac{1}{H_{0}}\right) \\
& \cong 4.408143 \times 10^{17} \mathrm{sec} \cong 13.969 \text { Billion years }
\end{aligned}
$$

According to standard cosmology, cosmic age corresponding to a temperature of $\sim 3000 \mathrm{~K}$ and redshift of $\sim 1100$ is around $\sim 3,80,000$ years. Based on our proposed assumptions, If $H_{t} \cong 2.47323 \times 10^{-12} \mathrm{sec}^{-1}$, obtained $T_{t} \cong 2991.9 \mathrm{~K} \cong 3000 \mathrm{~K}$ Corresponding cosmic age can be fitted with assumption $3 \mathrm{a}$ in the following way.

$$
t_{T \approx 2992 K} \cong\left[1+\ln \left(\frac{H_{T \approx 2992 K}}{H_{0}}\right)^{2}\right] \frac{1}{H_{T \approx 2992 K}} \cong 3,69,047 \text { years. }
$$

\begin{tabular}{|c|c|c|c|c|c|c|}
\hline $\begin{array}{l}\text { Assumed } \\
\text { Hubble } \\
\text { parameter } \\
(1 / \mathrm{sec})\end{array}$ & $\begin{array}{c}\begin{array}{c}\text { Estimated } \\
\text { factor }\end{array} \\
\sqrt{1+\ln \left(\frac{H_{p l}}{H_{t}}\right)}\end{array}$ & $\begin{array}{l}\text { Estimated } \\
\text { cosmic } \\
\text { temperature } \\
(\mathrm{K})\end{array}$ & $\begin{array}{c}\text { Estimated } \\
\text { Cosmic } \\
\text { age } \\
(\mathrm{sec})\end{array}$ & $\begin{array}{c}\text { Estimated } \\
\text { expansion } \\
\text { speed } \\
(\mathrm{m} / \mathrm{sec})\end{array}$ & $\begin{array}{l}\text { Estimated } \\
\text { cosmic } \\
\text { radius } \\
\text { (m) }\end{array}$ & $\begin{array}{c}\text { Estimated } \\
\text { cosmic } \\
\text { mass } \\
(\mathrm{kg})\end{array}$ \\
\hline $2.269 \mathrm{E}-18$ & 11.8851 & $2.72 \mathrm{E}+00$ & $4.41 \mathrm{E}+17$ & $3.56 \mathrm{E}+09$ & $8.514 \mathrm{E}+26$ & $1.147 \mathrm{E}+54$ \\
\hline $2.269 \mathrm{E}-17$ & 11.7879 & $8.67 \mathrm{E}+00$ & $2.47 \mathrm{E}+17$ & $3.53 \mathrm{E}+09$ & $4.736 \mathrm{E}+26$ & $6.378 \mathrm{E}+53$ \\
\hline $2.269 \mathrm{E}-16$ & 11.6898 & $2.77 \mathrm{E}+01$ & $4.50 \mathrm{E}+16$ & $3.50 \mathrm{E}+09$ & $8.561 \mathrm{E}+25$ & $1.153 \mathrm{E}+53$ \\
\hline $2.269 \mathrm{E}-15$ & 11.5909 & $8.82 \mathrm{E}+01$ & $6.53 \mathrm{E}+15$ & $3.47 \mathrm{E}+09$ & $1.233 \mathrm{E}+25$ & $1.660 \mathrm{E}+52$ \\
\hline $2.269 \mathrm{E}-14$ & 11.4911 & $2.81 \mathrm{E}+02$ & $8.56 \mathrm{E}+14$ & $3.44 \mathrm{E}+09$ & $1.603 \mathrm{E}+24$ & $2.159 \mathrm{E}+51$ \\
\hline $2.269 \mathrm{E}-13$ & 11.3905 & $8.98 \mathrm{E}+02$ & $1.06 \mathrm{E}+14$ & $3.41 \mathrm{E}+09$ & $1.967 \mathrm{E}+23$ & $2.649 \mathrm{E}+50$ \\
\hline $2.269 \mathrm{E}-12$ & 11.2890 & $2.86 \mathrm{E}+03$ & $1.26 \mathrm{E}+13$ & $3.38 \mathrm{E}+09$ & $2.325 \mathrm{E}+22$ & $3.131 \mathrm{E}+49$ \\
\hline $2.473 \mathrm{E}-12$ & 11.2851 & $2.99 \mathrm{E}+03$ & $1.16 \mathrm{E}+13$ & $3.38 \mathrm{E}+09$ & $2.145 \mathrm{E}+22$ & $2.888 \mathrm{E}+49$ \\
\hline $2.269 \mathrm{E}-11$ & 11.1865 & $9.14 \mathrm{E}+03$ & $1.47 \mathrm{E}+12$ & $3.35 \mathrm{E}+09$ & $2.676 \mathrm{E}+21$ & $3.604 \mathrm{E}+48$ \\
\hline $2.269 \mathrm{E}-10$ & 11.0831 & $2.92 \mathrm{E}+04$ & $1.67 \mathrm{E}+11$ & $3.32 \mathrm{E}+09$ & $3.021 \mathrm{E}+20$ & $4.069 \mathrm{E}+47$ \\
\hline $2.269 \mathrm{E}-09$ & 10.9788 & $9.31 \mathrm{E}+04$ & $1.87 \mathrm{E}+10$ & $3.29 \mathrm{E}+09$ & $3.360 \mathrm{E}+19$ & $4.524 \mathrm{E}+46$ \\
\hline $2.269 \mathrm{E}-08$ & 10.8734 & $2.97 \mathrm{E}+05$ & $2.07 \mathrm{E}+09$ & $3.26 \mathrm{E}+09$ & $3.691 \mathrm{E}+18$ & $4.971 \mathrm{E}+45$ \\
\hline $2.269 \mathrm{E}-07$ & 10.7670 & $9.50 \mathrm{E}+05$ & $2.28 \mathrm{E}+08$ & $3.23 \mathrm{E}+09$ & $4.016 \mathrm{E}+17$ & $5.409 \mathrm{E}+44$ \\
\hline $2.269 \mathrm{E}-06$ & 10.6595 & $3.03 \mathrm{E}+06$ & $2.48 \mathrm{E}+07$ & $3.20 \mathrm{E}+09$ & $4.335 \mathrm{E}+16$ & $5.837 \mathrm{E}+43$ \\
\hline $2.269 \mathrm{E}-05$ & 10.5510 & $9.69 \mathrm{E}+06$ & $2.68 \mathrm{E}+06$ & $3.16 \mathrm{E}+09$ & $4.646 \mathrm{E}+15$ & $6.256 \mathrm{E}+42$ \\
\hline
\end{tabular}

See the following table -1 .

Table-1: Various cosmic physical parameters 


\begin{tabular}{|c|c|c|c|c|c|c|}
\hline $2.269 \mathrm{E}-04$ & 10.4413 & $3.10 \mathrm{E}+07$ & $2.89 \mathrm{E}+05$ & $3.13 \mathrm{E}+09$ & $4.950 \mathrm{E}+14$ & $6.665 \mathrm{E}+41$ \\
\hline $2.269 \mathrm{E}-03$ & 10.3304 & $9.90 \mathrm{E}+07$ & $3.09 \mathrm{E}+04$ & $3.10 \mathrm{E}+09$ & $5.247 \mathrm{E}+13$ & $7.065 \mathrm{E}+40$ \\
\hline $2.269 \mathrm{E}-02$ & 10.2184 & $3.16 \mathrm{E}+08$ & $3.29 \mathrm{E}+03$ & $3.06 \mathrm{E}+09$ & $5.536 \mathrm{E}+12$ & $7.455 \mathrm{E}+39$ \\
\hline 2.269E-01 & 10.1051 & $1.01 \mathrm{E}+09$ & $3.50 \mathrm{E}+02$ & $3.03 \mathrm{E}+09$ & $5.818 \mathrm{E}+11$ & $7.835 \mathrm{E}+38$ \\
\hline $2.269 \mathrm{E}+00$ & 9.9905 & $3.24 \mathrm{E}+09$ & $3.70 \mathrm{E}+01$ & $3.00 \mathrm{E}+09$ & $6.092 \mathrm{E}+10$ & $8.204 \mathrm{E}+37$ \\
\hline $2.269 \mathrm{E}+01$ & 9.8746 & $1.04 \mathrm{E}+10$ & $3.90 \mathrm{E}+00$ & $2.96 \mathrm{E}+09$ & $6.359 \mathrm{E}+09$ & $8.563 \mathrm{E}+36$ \\
\hline $2.269 \mathrm{E}+02$ & 9.7573 & $3.31 \mathrm{E}+10$ & $4.10 \mathrm{E}-01$ & $2.93 \mathrm{E}+09$ & $6.618 \mathrm{E}+08$ & $8.912 \mathrm{E}+35$ \\
\hline $2.269 \mathrm{E}+03$ & 9.6386 & $1.06 \mathrm{E}+11$ & $4.31 \mathrm{E}-02$ & $2.89 \mathrm{E}+09$ & $6.869 \mathrm{E}+07$ & $9.249 \mathrm{E}+34$ \\
\hline $2.269 \mathrm{E}+04$ & 9.5184 & $3.40 \mathrm{E}+11$ & $4.51 \mathrm{E}-03$ & $2.85 \mathrm{E}+09$ & $7.111 \mathrm{E}+06$ & $9.576 \mathrm{E}+33$ \\
\hline $2.269 \mathrm{E}+05$ & 9.3966 & $1.09 \mathrm{E}+12$ & 4.71E-04 & $2.82 \mathrm{E}+09$ & $7.345 \mathrm{E}+05$ & $9.891 \mathrm{E}+32$ \\
\hline $2.269 \mathrm{E}+06$ & 9.2733 & $3.49 \mathrm{E}+12$ & $4.92 \mathrm{E}-05$ & $2.78 \mathrm{E}+09$ & $7.571 \mathrm{E}+04$ & $1.019 \mathrm{E}+32$ \\
\hline $2.269 \mathrm{E}+07$ & 9.1483 & $1.12 \mathrm{E}+13$ & $5.12 \mathrm{E}-06$ & $2.74 \mathrm{E}+09$ & $7.787 \mathrm{E}+03$ & $1.049 \mathrm{E}+31$ \\
\hline $2.269 \mathrm{E}+08$ & 9.0216 & $3.58 \mathrm{E}+13$ & 5.32E-07 & $2.70 \mathrm{E}+09$ & $7.995 \mathrm{E}+02$ & $1.077 \mathrm{E}+30$ \\
\hline $2.269 \mathrm{E}+09$ & 8.8931 & $1.15 \mathrm{E}+14$ & $5.53 \mathrm{E}-08$ & $2.67 \mathrm{E}+09$ & $8.193 \mathrm{E}+01$ & $1.103 E+29$ \\
\hline $2.269 \mathrm{E}+10$ & 8.7626 & $3.69 \mathrm{E}+14$ & 5.73E-09 & $2.63 \mathrm{E}+09$ & $8.382 \mathrm{E}+00$ & $1.129 \mathrm{E}+28$ \\
\hline $2.269 \mathrm{E}+11$ & 8.6303 & $1.18 \mathrm{E}+15$ & $5.93 \mathrm{E}-10$ & $2.59 \mathrm{E}+09$ & $8.562 \mathrm{E}-01$ & $1.153 \mathrm{E}+27$ \\
\hline $2.269 \mathrm{E}+12$ & 8.4958 & $3.81 \mathrm{E}+15$ & $6.13 \mathrm{E}-11$ & $2.55 \mathrm{E}+09$ & $8.731 \mathrm{E}-02$ & $1.176 \mathrm{E}+26$ \\
\hline $2.269 \mathrm{E}+13$ & 8.3592 & $1.22 \mathrm{E}+16$ & $6.34 \mathrm{E}-12$ & $2.51 \mathrm{E}+09$ & 8.890E-03 & $1.197 \mathrm{E}+25$ \\
\hline $2.269 \mathrm{E}+14$ & 8.2203 & $3.93 \mathrm{E}+16$ & $6.54 \mathrm{E}-13$ & $2.46 \mathrm{E}+09$ & $9.039 \mathrm{E}-04$ & $1.217 \mathrm{E}+24$ \\
\hline $2.269 \mathrm{E}+15$ & 8.0791 & $1.27 \mathrm{E}+17$ & $6.74 \mathrm{E}-14$ & $2.42 \mathrm{E}+09$ & $9.177 \mathrm{E}-05$ & $1.236 \mathrm{E}+23$ \\
\hline $2.269 \mathrm{E}+16$ & 7.9353 & $4.08 \mathrm{E}+17$ & $6.95 \mathrm{E}-15$ & $2.38 \mathrm{E}+09$ & $9.303 \mathrm{E}-06$ & $1.253 \mathrm{E}+22$ \\
\hline $2.269 \mathrm{E}+17$ & 7.7888 & $1.31 \mathrm{E}+18$ & $7.15 \mathrm{E}-16$ & +09 & $9.418 \mathrm{E}-07$ & $1.268 \mathrm{E}+21$ \\
\hline $2.269 \mathrm{E}+18$ & 7.6396 & $4.23 \mathrm{E}+18$ & $7.35 \mathrm{E}-17$ & $2.29 \mathrm{E}+09$ & $9.521 \mathrm{E}-08$ & $1.282 \mathrm{E}+20$ \\
\hline $2.269 \mathrm{E}+19$ & 7.4874 & $1.37 \mathrm{E}+19$ & $7.56 \mathrm{E}-18$ & $2.24 \mathrm{E}+09$ & 9.612E-09 & $1.294 \mathrm{E}+19$ \\
\hline $2.269 \mathrm{E}+20$ & 7.3320 & $4.41 \mathrm{E}+19$ & $7.76 \mathrm{E}-19$ & $2.20 \mathrm{E}+09$ & $9.689 \mathrm{E}-10$ & $1.305 \mathrm{E}+18$ \\
\hline $2.269 \mathrm{E}+21$ & 7.1733 & $1.43 \mathrm{E}+20$ & $7.96 \mathrm{E}-20$ & $2.15 \mathrm{E}+09$ & $9.754 \mathrm{E}-11$ & $1.313 \mathrm{E}+17$ \\
\hline $2.269 \mathrm{E}+22$ & 7.0109 & $4.61 \mathrm{E}+20$ & $8.16 \mathrm{E}-21$ & $2.10 \mathrm{E}+09$ & $9.804 \mathrm{E}-12$ & $1.320 \mathrm{E}+16$ \\
\hline $2.269 \mathrm{E}+23$ & 6.8447 & $1.49 \mathrm{E}+21$ & $8.37 \mathrm{E}-22$ & $2.05 \mathrm{E}+09$ & $9.839 \mathrm{E}-13$ & $1.325 \mathrm{E}+15$ \\
\hline $2.269 \mathrm{E}+24$ & 6.6744 & $4.84 \mathrm{E}+21$ & $8.57 \mathrm{E}-23$ & $2.00 \mathrm{E}+09$ & $9.859 \mathrm{E}-14$ & $1.328 \mathrm{E}+14$ \\
\hline $2.269 \mathrm{E}+25$ & 6.4996 & $1.57 \mathrm{E}+22$ & $8.77 \mathrm{E}-24$ & $1.95 \mathrm{E}+09$ & $9.863 \mathrm{E}-15$ & $1.328 \mathrm{E}+13$ \\
\hline $2.269 \mathrm{E}+26$ & 6.3200 & $5.12 \mathrm{E}+22$ & $8.98 \mathrm{E}-25$ & $1.89 \mathrm{E}+09$ & $9.849 \mathrm{E}-16$ & $1.326 \mathrm{E}+12$ \\
\hline $2.269 \mathrm{E}+27$ & 6.1352 & $1.67 \mathrm{E}+23$ & $9.18 \mathrm{E}-26$ & $1.84 \mathrm{E}+09$ & $9.817 \mathrm{E}-17$ & $1.322 \mathrm{E}+11$ \\
\hline $2.269 \mathrm{E}+28$ & 5.9445 & $5.44 \mathrm{E}+23$ & $9.38 \mathrm{E}-27$ & $1.78 \mathrm{E}+09$ & $9.766 \mathrm{E}-18$ & $1.315 \mathrm{E}+10$ \\
\hline $2.269 \mathrm{E}+29$ & 5.7476 & $1.78 \mathrm{E}+24$ & $9.59 \mathrm{E}-28$ & $1.72 \mathrm{E}+09$ & $9.695 \mathrm{E}-19$ & $1.306 \mathrm{E}+09$ \\
\hline $2.269 \mathrm{E}+30$ & 5.5437 & $5.83 \mathrm{E}+24$ & $9.79 \mathrm{E}-29$ & $1.66 \mathrm{E}+09$ & $9.601 \mathrm{E}-20$ & $1.293 \mathrm{E}+08$ \\
\hline $2.269 \mathrm{E}+31$ & 5.3320 & $1.92 \mathrm{E}+25$ & $9.99 \mathrm{E}-30$ & $1.60 \mathrm{E}+09$ & $9.483 \mathrm{E}-21$ & $1.277 \mathrm{E}+07$ \\
\hline $2.269 \mathrm{E}+32$ & 5.1115 & $6.33 \mathrm{E}+25$ & $1.02 \mathrm{E}-30$ & $1.53 \mathrm{E}+09$ & 9.339E-22 & $1.258 \mathrm{E}+06$ \\
\hline $2.269 \mathrm{E}+33$ & 4.8810 & $2.09 \mathrm{E}+26$ & $1.04 \mathrm{E}-31$ & $1.46 \mathrm{E}+09$ & $9.166 \mathrm{E}-23$ & $1.234 \mathrm{E}+05$ \\
\hline $2.269 \mathrm{E}+34$ & 4.6392 & $6.97 \mathrm{E}+26$ & $1.06 \mathrm{E}-32$ & $1.39 \mathrm{E}+09$ & $8.960 \mathrm{E}-24$ & $1.207 \mathrm{E}+04$ \\
\hline $2.269 \mathrm{E}+35$ & 4.3840 & $2.33 \mathrm{E}+27$ & $1.08 \mathrm{E}-33$ & $1.31 \mathrm{E}+09$ & $8.719 \mathrm{E}-25$ & $1.174 \mathrm{E}+03$ \\
\hline $2.269 \mathrm{E}+36$ & 4.1130 & $7.86 \mathrm{E}+27$ & $1.10 \mathrm{E}-34$ & $1.23 \mathrm{E}+09$ & $8.435 \mathrm{E}-26$ & $1.136 \mathrm{E}+02$ \\
\hline
\end{tabular}




\begin{tabular}{|l|l|l|l|l|l|l|}
$2.269 \mathrm{E}+37$ & 3.8229 & $2.67 \mathrm{E}+28$ & $1.12 \mathrm{E}-35$ & $1.15 \mathrm{E}+09$ & $8.103 \mathrm{E}-27$ & $1.091 \mathrm{E}+01$ \\
\hline $2.269 \mathrm{E}+38$ & 3.5088 & $9.22 \mathrm{E}+28$ & $1.14 \mathrm{E}-36$ & $1.05 \mathrm{E}+09$ & $7.713 \mathrm{E}-28$ & $1.039 \mathrm{E}+00$ \\
\hline $2.269 \mathrm{E}+39$ & 3.1637 & $3.23 \mathrm{E}+29$ & $1.16 \mathrm{E}-37$ & $9.48 \mathrm{E}+08$ & $7.249 \mathrm{E}-29$ & $9.762 \mathrm{E}-02$ \\
\hline $2.269 \mathrm{E}+40$ & 2.7761 & $1.16 \mathrm{E}+30$ & $1.18 \mathrm{E}-38$ & $8.32 \mathrm{E}+08$ & $6.689 \mathrm{E}-30$ & $9.008 \mathrm{E}-03$ \\
\hline $2.269 \mathrm{E}+41$ & 2.3246 & $4.40 \mathrm{E}+30$ & $1.20 \mathrm{E}-39$ & $6.97 \mathrm{E}+08$ & $5.991 \mathrm{E}-31$ & $8.067 \mathrm{E}-04$ \\
\hline $2.269 \mathrm{E}+42$ & 1.7610 & $1.84 \mathrm{E}+31$ & $1.22 \mathrm{E}-40$ & $5.28 \mathrm{E}+08$ & $5.059 \mathrm{E}-32$ & $6.813 \mathrm{E}-05$ \\
\hline
\end{tabular}

\section{Application-3: To understand the cosmic scale factor with reference to $(z+1)$}

According to modern cosmology, cosmic age connected with cosmic scale factor is given by,

$$
t \cong\left(\frac{1}{z+1}\right)^{\frac{3}{2}}\left(\frac{1}{H_{0}}\right)
$$

where $\left(\frac{a_{\text {now }}}{a_{\text {then }}}\right) \cong(z+1)$ is the scale factor. With reference to our proposed assumption 3a, cosmic scale factor can be expressed with the following relation.

$$
(z+1) \cong\left(\frac{H_{t}}{H_{0}}\right)^{\frac{2}{3}}\left[1+\ln \left(\frac{H_{t}}{H_{0}}\right)^{2}\right]^{-2 / 3}
$$

For the CMBR redshift case, i.e. $\quad T_{t} \cong 3000 \mathrm{~K}$ and $z \cong 1100$;

$$
H_{t} \cong 2.47323 \times 10^{-12} \sec ^{-1} \cong H_{z \approx 1100} \cdots(\text { say })
$$

Estimated, $T_{t} \cong 2991.9 \mathrm{~K} \approx T_{z \approx 1100} \ldots . . .($ say $)$

Corresponding $(z+1)$ can be fitted with the following relation.

$$
(z+1) \cong\left(\frac{H_{z \approx 1100}}{H_{0}}\right)^{\frac{2}{3}}\left[1+\ln \left(\frac{H_{z \approx 1100}}{H_{0}}\right)^{2}\right]^{-2 / 3} \cong 1127.32
$$

With further study, it is also possible to show that,

$$
\begin{aligned}
& (z+1)^{3 / 2} \cong \frac{V_{0} t_{0}}{V_{t} t} \cong \frac{R_{0}}{R_{t}} \\
& \rightarrow R_{t} \cong\left(\frac{V_{0} t_{0}}{V_{t} t_{t}}\right) R_{0} \cong(z+1)^{-3 / 2} R_{0} \\
& \Rightarrow \frac{R_{t}}{R_{0}} \cong(z+1)^{-3 / 2} \text { Or }(\mathrm{z}+1) \cong\left(\frac{R_{0}}{R_{t}}\right)^{\frac{2}{3}}
\end{aligned}
$$


where $V_{0}$ and $V_{t}$ represent the current and past cosmic expansion speeds, $t_{0}$ and $t$ represent current and past cosmic ages and $R_{0}$ and $R_{t}$ represent current and past cosmic radii.

$$
(z+1) \approx\left(\frac{H_{t}}{H_{0}}\right)^{2 / 3}\left[1+\ln \left(\frac{H_{t}}{H_{0}}\right)^{2}\right]^{-2 / 3} \approx\left(\frac{V_{0} * t_{0}}{V_{t} * t}\right)^{2 / 3}
$$

\section{Application-4: To understand the cosmic scale factor with reference to $\left(T_{t} / T_{0}\right)$}

According to modern cosmology, cosmic age connected with cosmic temperature ratio is given by,

$$
(z+1) \cong \frac{T_{t}}{T_{0}}
$$

With reference to the data prepared as per the proposed relations, from relations (2) and (4) it is possible to show that,

$$
\left.\begin{array}{l}
\text { Case-A) }\left\{(z+1) \cong \frac{T_{t}}{T_{0}}\right\} \cong \sqrt{\left(\frac{V_{0}^{2}}{H_{0}}\right) /\left(\frac{V_{t}^{2}}{H_{t}}\right)} \ldots \ldots \ldots . .(17 \mathrm{~A}) \\
\text { Case-B) }\left\{(z+1) \cong \frac{T_{t}}{T_{0}}\right\} \approx \sqrt{\left(\frac{V_{0}}{H_{0}}\right) /\left(\frac{V_{t}}{H_{t}}\right)} \ldots \ldots \ldots . .(17 \mathrm{~B})
\end{array}\right\}
$$

where $V_{0}$ and $V_{t}$ represent the current and past cosmic expansion speeds respectively. Point to be noted is that, magnitude of $\left(\frac{V_{t}^{2}}{H_{t}}\right)$ and $\left(\frac{V_{t}}{H_{t}}\right)$ increase with cosmic age.

For the CMBR redshift case, i.e. $\quad T_{t} \cong 3000 \mathrm{~K}$ and $z \cong 1100$;

$H_{t} \cong 2.47323 \times 10^{-12} \sec ^{-1} \cong H_{z \approx 1100} \cdots($ say $)$

Estimated, $T_{t} \cong 2991.9 \mathrm{~K} \approx T_{z \approx 1100} \ldots . .($ say $)$

Estimated, $V_{t} \cong 3.3832 \times 10^{9} \mathrm{~m} \cdot \mathrm{sec}^{-1} \approx V_{z \approx 1100} \cdots . .($ say $)$

For case-A, it is noticed that,

Estimated, $\frac{V_{t}^{2}}{H_{t}} \cong 4.62798 \times 10^{30} \mathrm{~m}^{2} \cdot \mathrm{sec}^{-1} \cong \frac{V_{z \approx 1100}^{2}}{H_{z \approx 1100}} \ldots . .($ say $)$

Estimated, $\frac{V_{0}^{2}}{H_{0}} \cong 5.59635 \times 10^{36} \mathrm{~m}^{2} \cdot \mathrm{sec}^{-1}$

It is noticed that,

$$
(z+1) \cong \sqrt{\left(\frac{V_{0}^{2}}{H_{0}}\right) /\left(\frac{V_{z \approx 1100}^{2}}{H_{z \approx 1100}}\right)} \cong 1099.6
$$


Point to be noted is that, dimensionally, $\left(\frac{V_{t}^{2}}{H_{t}}\right)$ corresponds to 'length $2 /$ meter' and $(z+1)^{2}$ seems to represent a ratio of $\left(\frac{V_{0}^{2}}{H_{0}}\right) /\left(\frac{V_{t}^{2}}{H_{t}}\right)$.

For case-B, it is noticed that,

Estimated, $\frac{V_{t}}{H_{t}} \cong 1.36793 \times 10^{21} \mathrm{~m} \cong \frac{V_{z}}{H_{z \approx 1100}} \ldots . . .($ say $)$

Estimated, $\frac{V_{0}}{H_{0}} \cong 1.57065 \times 10^{27} \mathrm{~m}$

$$
(z+1) \cong \sqrt{\left(\frac{V_{0}}{H_{0}}\right) /\left(\frac{V_{z}}{H_{z \approx 1100}}\right)} \cong 1071.54
$$

Point to be noted is that, dimensionally, $\left(\frac{V_{t}}{H_{t}}\right)$ corresponds to 'length' and $(z+1)^{2}$ seems to represent a ratio of $\left(\frac{V_{0}}{H 0_{t}}\right) /\left(\frac{V_{t}}{H_{t}}\right)$.

It may be noted that, relations [(12) and (15)] and [(17A) or (17B)] start deviating when temperature reaches the order of $3000 \mathrm{~K}$. With respect to radiation dominated era and matter dominated era above relations can be recommended for further research. See the following figure- 2 and table -2 for various estimated redshift magnitudes.

Figure 2: Comparison of relations $(17 \mathrm{~A}, 17 \mathrm{~B})$ and $(12,14)$

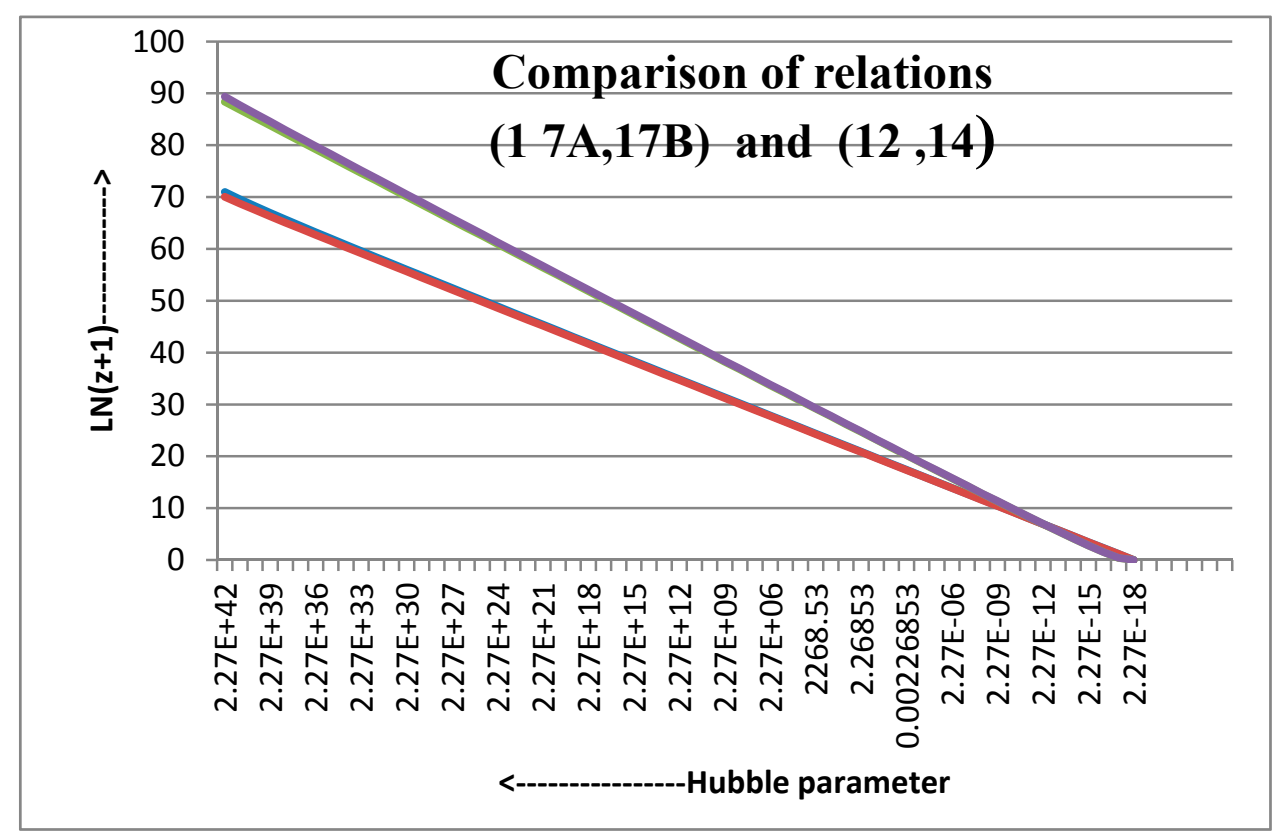

In this figure-2, bottom two lines represent the natural logarithm of relations (17A and 17B) and top two lines represent the natural logarithm of relations (12 and 14). 
Table-2: To estimate various redshift magnitudes connected with cosmic age and radius

\begin{tabular}{|c|c|c|c|c|c|c|c|c|}
\hline $\begin{array}{c}\text { Assumed } \\
\text { Hubble } \\
\text { parameter } \\
(1 / \mathrm{sec})\end{array}$ & $\begin{array}{c}\text { Estimated } \\
\text { cosmic } \\
\text { temperatu } \\
\text { re } \\
(\mathrm{K})\end{array}$ & $\begin{array}{c}\text { Estimate } \\
\text { d Cosmic } \\
\text { age } \\
(\mathrm{sec})\end{array}$ & $\begin{array}{c}\text { Estimate } \\
\text { d } \\
\text { expansio } \\
\text { n speed } \\
(\mathrm{m} / \mathrm{sec})\end{array}$ & $\begin{array}{l}\text { Estimated } \\
\text { cosmic } \\
\text { radius } \\
\text { (m) }\end{array}$ & $\begin{array}{c}(\mathrm{z}+1) \\
\text { from } \\
\text { relation } \\
(12)\end{array}$ & $\begin{array}{c}(\mathrm{z}+1) \text { from } \\
\text { relation } \\
(15)\end{array}$ & $\begin{array}{c}(\mathrm{z}+1) \\
\text { from } \\
\text { relation } \\
(17 \mathrm{~A})\end{array}$ & $\begin{array}{l}(\mathrm{z}+1) \\
\text { from } \\
\text { relation } \\
(17 \mathrm{~B})\end{array}$ \\
\hline $2.269 \mathrm{E}-18$ & $2.72 \mathrm{E}+00$ & $4.41 \mathrm{E}+17$ & $3.56 \mathrm{E}+09$ & $8.514 \mathrm{E}+26$ & $1.000 \mathrm{E}+00$ & $1.000 \mathrm{E}+00$ & $1.000 \mathrm{E}+00$ & $1.000 \mathrm{E}+00$ \\
\hline $2.269 \mathrm{E}-17$ & $8.67 \mathrm{E}+00$ & $2.47 \mathrm{E}+17$ & $3.53 \mathrm{E}+09$ & $4.736 \mathrm{E}+26$ & $1.471 \mathrm{E}+00$ & $1.479 \mathrm{E}+00$ & $3.188 \mathrm{E}+00$ & $3.175 \mathrm{E}+00$ \\
\hline $2.269 \mathrm{E}-16$ & $2.77 \mathrm{E}+01$ & $4.50 \mathrm{E}+16$ & $3.50 \mathrm{E}+09$ & $8.561 \mathrm{E}+25$ & $4.578 \mathrm{E}+00$ & $4.628 \mathrm{E}+00$ & $1.017 \mathrm{E}+01$ & $1.008 \mathrm{E}+01$ \\
\hline $2.269 \mathrm{E}-15$ & $8.82 \mathrm{E}+01$ & $6.53 \mathrm{E}+15$ & $3.47 \mathrm{E}+09$ & $1.233 \mathrm{E}+25$ & $1.658 \mathrm{E}+01$ & $1.686 \mathrm{E}+01$ & $3.243 \mathrm{E}+01$ & $3.202 \mathrm{E}+01$ \\
\hline $2.269 \mathrm{E}-14$ & $2.81 \mathrm{E}+02$ & $8.56 \mathrm{E}+14$ & $3.44 \mathrm{E}+09$ & $1.603 \mathrm{E}+24$ & $6.424 \mathrm{E}+01$ & $6.570 \mathrm{E}+01$ & $1.034 \mathrm{E}+02$ & $1.017 \mathrm{E}+02$ \\
\hline $2.269 \mathrm{E}-13$ & $8.98 \mathrm{E}+02$ & $1.06 \mathrm{E}+14$ & $3.41 \mathrm{E}+09$ & $1.967 \mathrm{E}+23$ & $2.588 \mathrm{E}+02$ & $2.662 \mathrm{E}+02$ & $3.300 \mathrm{E}+02$ & $3.230 \mathrm{E}+02$ \\
\hline $2.269 \mathrm{E}-12$ & $2.86 \mathrm{E}+03$ & $1.26 \mathrm{E}+13$ & $3.38 \mathrm{E}+09$ & $2.325 \mathrm{E}+22$ & $1.069 \mathrm{E}+03$ & $1.106 \mathrm{E}+03$ & $1.053 \mathrm{E}+03$ & $1.026 \mathrm{E}+03$ \\
\hline $2.473 E-12$ & $2.99 \mathrm{E}+03$ & $1.16 \mathrm{E}+13$ & $3.38 \mathrm{E}+09$ & $2.145 \mathrm{E}+22$ & $1.127 E+03$ & $1.167 E+03$ & $1.100 \mathrm{E}+03$ & $1.072 E+03$ \\
\hline $2.269 \mathrm{E}-11$ & $9.14 \mathrm{E}+03$ & $1.47 \mathrm{E}+12$ & $3.35 \mathrm{E}+09$ & $2.676 \mathrm{E}+21$ & $4.490 \mathrm{E}+03$ & $4.675 \mathrm{E}+03$ & $3.360 \mathrm{E}+03$ & $3.260 \mathrm{E}+03$ \\
\hline $2.269 \mathrm{E}-10$ & $2.92 \mathrm{E}+04$ & $1.67 \mathrm{E}+11$ & $3.32 \mathrm{E}+09$ & $3.021 \mathrm{E}+20$ & $1.911 \mathrm{E}+04$ & $2.003 \mathrm{E}+04$ & $1.072 \mathrm{E}+04$ & $1.036 \mathrm{E}+04$ \\
\hline $2.269 \mathrm{E}-09$ & $9.31 \mathrm{E}+04$ & $1.87 \mathrm{E}+10$ & $3.29 \mathrm{E}+09$ & $3.360 \mathrm{E}+19$ & $8.218 \mathrm{E}+04$ & $8.664 \mathrm{E}+04$ & $3.423 \mathrm{E}+04$ & $3.290 \mathrm{E}+04$ \\
\hline $2.269 \mathrm{E}-08$ & $2.97 \mathrm{E}+05$ & $2.07 \mathrm{E}+09$ & $3.26 \mathrm{E}+09$ & $3.691 \mathrm{E}+18$ & $3.561 \mathrm{E}+05$ & $3.779 \mathrm{E}+05$ & $1.093 \mathrm{E}+05$ & $1.045 \mathrm{E}+05$ \\
\hline $2.269 \mathrm{E}-07$ & $9.50 \mathrm{E}+05$ & $2.28 \mathrm{E}+08$ & $3.23 \mathrm{E}+09$ & $4.016 \mathrm{E}+17$ & $1.553 \mathrm{E}+06$ & $1.659 \mathrm{E}+06$ & $3.491 \mathrm{E}+05$ & $3.322 \mathrm{E}+05$ \\
\hline $2.269 \mathrm{E}-06$ & $3.03 \mathrm{E}+06$ & $2.48 \mathrm{E}+07$ & $3.20 \mathrm{E}+09$ & $4.335 \mathrm{E}+16$ & $6.811 \mathrm{E}+06$ & $7.323 \mathrm{E}+06$ & $1.115 \mathrm{E}+06$ & $1.056 \mathrm{E}+06$ \\
\hline $2.269 \mathrm{E}-05$ & $9.69 \mathrm{E}+06$ & $2.68 \mathrm{E}+06$ & $3.16 \mathrm{E}+09$ & $4.646 \mathrm{E}+15$ & $3.000 \mathrm{E}+07$ & $3.248 \mathrm{E}+07$ & $3.562 \mathrm{E}+06$ & $3.356 \mathrm{E}+06$ \\
\hline $2.269 \mathrm{E}-04$ & $3.10 \mathrm{E}+07$ & $2.89 \mathrm{E}+05$ & $3.13 \mathrm{E}+09$ & $4.950 \mathrm{E}+14$ & $1.326 \mathrm{E}+08$ & $1.446 \mathrm{E}+08$ & $1.138 \mathrm{E}+07$ & $1.067 \mathrm{E}+07$ \\
\hline $2.269 \mathrm{E}-03$ & $9.90 \mathrm{E}+07$ & $3.09 \mathrm{E}+04$ & $3.10 \mathrm{E}+09$ & $5.247 \mathrm{E}+13$ & $5.883 \mathrm{E}+08$ & $6.460 \mathrm{E}+08$ & $3.638 \mathrm{E}+07$ & $3.392 \mathrm{E}+07$ \\
\hline $2.269 \mathrm{E}-02$ & $3.16 \mathrm{E}+08$ & $3.29 \mathrm{E}+03$ & $3.06 \mathrm{E}+09$ & $5.536 \mathrm{E}+12$ & $2.617 \mathrm{E}+09$ & $2.895 \mathrm{E}+09$ & $1.163 \mathrm{E}+08$ & $1.078 \mathrm{E}+08$ \\
\hline $2.269 \mathrm{E}-01$ & $1.01 \mathrm{E}+09$ & $3.50 \mathrm{E}+02$ & $3.03 \mathrm{E}+09$ & $5.818 \mathrm{E}+11$ & $1.167 \mathrm{E}+10$ & $1.301 \mathrm{E}+10$ & $3.719 \mathrm{E}+08$ & $3.430 \mathrm{E}+08$ \\
\hline $2.269 \mathrm{E}+00$ & $3.24 \mathrm{E}+09$ & $3.70 \mathrm{E}+01$ & $3.00 \mathrm{E}+09$ & $6.092 \mathrm{E}+10$ & $5.218 \mathrm{E}+10$ & $5.859 \mathrm{E}+10$ & $1.190 \mathrm{E}+09$ & $1.091 \mathrm{E}+09$ \\
\hline $2.269 \mathrm{E}+01$ & $1.04 \mathrm{E}+10$ & $3.90 \mathrm{E}+00$ & $2.96 \mathrm{E}+09$ & $6.359 \mathrm{E}+09$ & $2.337 \mathrm{E}+11$ & $2.645 \mathrm{E}+11$ & $3.806 \mathrm{E}+09$ & $3.469 \mathrm{E}+09$ \\
\hline $2.269 \mathrm{E}+02$ & $3.31 \mathrm{E}+10$ & 4.10E-01 & $2.93 \mathrm{E}+09$ & $6.618 \mathrm{E}+08$ & $1.049 \mathrm{E}+12$ & $1.196 \mathrm{E}+12$ & $1.218 \mathrm{E}+10$ & $1.104 \mathrm{E}+10$ \\
\hline $2.269 \mathrm{E}+03$ & $1.06 \mathrm{E}+11$ & $4.31 \mathrm{E}-02$ & $2.89 \mathrm{E}+09$ & $6.869 \mathrm{E}+07$ & $4.714 \mathrm{E}+12$ & $5.420 \mathrm{E}+12$ & $3.899 \mathrm{E}+10$ & $3.512 \mathrm{E}+10$ \\
\hline $2.269 \mathrm{E}+04$ & $3.40 \mathrm{E}+11$ & $4.51 \mathrm{E}-03$ & $2.85 \mathrm{E}+09$ & $7.111 \mathrm{E}+06$ & $2.122 \mathrm{E}+13$ & $2.460 \mathrm{E}+13$ & $1.249 \mathrm{E}+11$ & $1.117 \mathrm{E}+11$ \\
\hline $2.269 \mathrm{E}+05$ & $1.09 \mathrm{E}+12$ & $4.71 \mathrm{E}-04$ & $2.82 \mathrm{E}+09$ & $7.345 \mathrm{E}+05$ & $9.564 \mathrm{E}+13$ & $1.119 \mathrm{E}+14$ & $4.000 \mathrm{E}+11$ & $3.556 \mathrm{E}+11$ \\
\hline $2.269 \mathrm{E}+06$ & $3.49 \mathrm{E}+12$ & 4.92E-05 & $2.78 \mathrm{E}+09$ & $7.571 \mathrm{E}+04$ & $4.316 \mathrm{E}+14$ & $5.093 \mathrm{E}+14$ & $1.282 \mathrm{E}+12$ & $1.132 \mathrm{E}+12$ \\
\hline $2.269 \mathrm{E}+07$ & $1.12 \mathrm{E}+13$ & $5.12 \mathrm{E}-06$ & $2.74 \mathrm{E}+09$ & $7.787 \mathrm{E}+03$ & $1.950 \mathrm{E}+15$ & $2.322 \mathrm{E}+15$ & $4.108 \mathrm{E}+12$ & $3.604 \mathrm{E}+12$ \\
\hline $2.269 \mathrm{E}+08$ & $3.58 \mathrm{E}+13$ & $5.32 \mathrm{E}-07$ & $2.70 \mathrm{E}+09$ & $7.995 \mathrm{E}+02$ & $8.820 \mathrm{E}+15$ & $1.060 \mathrm{E}+16$ & $1.317 \mathrm{E}+13$ & $1.148 \mathrm{E}+13$ \\
\hline $2.269 \mathrm{E}+09$ & $1.15 \mathrm{E}+14$ & $5.53 \mathrm{E}-08$ & $2.67 \mathrm{E}+09$ & $8.193 \mathrm{E}+01$ & $3.993 \mathrm{E}+16$ & $4.844 \mathrm{E}+16$ & $4.226 \mathrm{E}+13$ & $3.656 \mathrm{E}+13$ \\
\hline $2.269 \mathrm{E}+10$ & $3.69 \mathrm{E}+14$ & $5.73 \mathrm{E}-09$ & $2.63 \mathrm{E}+09$ & $8.382 \mathrm{E}+00$ & $1.809 \mathrm{E}+17$ & $2.217 \mathrm{E}+17$ & $1.356 \mathrm{E}+14$ & $1.165 \mathrm{E}+14$ \\
\hline $2.269 \mathrm{E}+11$ & $1.18 \mathrm{E}+15$ & $5.93 \mathrm{E}-10$ & $2.59 \mathrm{E}+09$ & $8.562 \mathrm{E}-01$ & $8.205 \mathrm{E}+17$ & $1.016 \mathrm{E}+18$ & $4.355 \mathrm{E}+14$ & $3.711 \mathrm{E}+14$ \\
\hline $2.269 \mathrm{E}+12$ & $3.81 \mathrm{E}+15$ & $6.13 \mathrm{E}-11$ & $2.55 \mathrm{E}+09$ & $8.731 \mathrm{E}-02$ & $3.724 \mathrm{E}+18$ & $4.658 \mathrm{E}+18$ & $1.399 \mathrm{E}+15$ & $1.183 \mathrm{E}+15$ \\
\hline $2.269 \mathrm{E}+13$ & $1.22 \mathrm{E}+16$ & $6.34 \mathrm{E}-12$ & $2.51 \mathrm{E}+09$ & $8.890 \mathrm{E}-03$ & $1.691 \mathrm{E}+19$ & $2.139 \mathrm{E}+19$ & $4.496 \mathrm{E}+15$ & $3.771 \mathrm{E}+15$ \\
\hline $2.269 \mathrm{E}+14$ & $3.93 \mathrm{E}+16$ & $6.54 \mathrm{E}-13$ & $2.46 \mathrm{E}+09$ & $9.039 \mathrm{E}-04$ & $7.687 \mathrm{E}+19$ & $9.829 \mathrm{E}+19$ & $1.446 \mathrm{E}+16$ & $1.202 \mathrm{E}+16$ \\
\hline $2.269 \mathrm{E}+15$ & $1.27 \mathrm{E}+17$ & $6.74 \mathrm{E}-14$ & $2.42 \mathrm{E}+09$ & $9.177 \mathrm{E}-05$ & $3.496 \mathrm{E}+20$ & $4.522 \mathrm{E}+20$ & $4.652 \mathrm{E}+16$ & $3.835 \mathrm{E}+16$ \\
\hline $2.269 \mathrm{E}+16$ & $4.08 \mathrm{E}+17$ & $6.95 \mathrm{E}-15$ & $2.38 \mathrm{E}+09$ & $9.303 \mathrm{E}-06$ & $1.591 \mathrm{E}+21$ & $2.083 \mathrm{E}+21$ & $1.498 \mathrm{E}+17$ & $1.224 \mathrm{E}+17$ \\
\hline
\end{tabular}




\begin{tabular}{|c|c|c|c|c|c|c|c|c|}
\hline $2.269 \mathrm{E}+17$ & $1.31 \mathrm{E}+18$ & $7.15 \mathrm{E}-16$ & $2.34 \mathrm{E}+09$ & $9.418 \mathrm{E}-07$ & $7.244 \mathrm{E}+21$ & $9.602 \mathrm{E}+21$ & $4.825 \mathrm{E}+17$ & $3.906 \mathrm{E}+17$ \\
\hline $2.269 \mathrm{E}+18$ & $4.23 \mathrm{E}+18$ & $7.35 \mathrm{E}-17$ & $2.29 \mathrm{E}+09$ & $9.521 \mathrm{E}-08$ & $3.300 \mathrm{E}+22$ & $4.431 \mathrm{E}+22$ & $1.556 \mathrm{E}+18$ & $1.247 \mathrm{E}+18$ \\
\hline $2.269 \mathrm{E}+19$ & $1.37 \mathrm{E}+19$ & $7.56 \mathrm{E}-18$ & $2.24 \mathrm{E}+09$ & $9.612 \mathrm{E}-09$ & $1.504 \mathrm{E}+23$ & $2.047 \mathrm{E}+23$ & $5.020 \mathrm{E}+18$ & $3.984 \mathrm{E}+18$ \\
\hline $2.269 \mathrm{E}+20$ & $4.41 \mathrm{E}+19$ & $7.76 \mathrm{E}-19$ & $2.20 \mathrm{E}+09$ & $9.689 \mathrm{E}-10$ & $6.860 \mathrm{E}+23$ & $9.466 \mathrm{E}+23$ & $1.621 \mathrm{E}+19$ & $1.273 \mathrm{E}+19$ \\
\hline $2.269 \mathrm{E}+21$ & $1.43 \mathrm{E}+20$ & $7.96 \mathrm{E}-20$ & $2.15 \mathrm{E}+09$ & $9.754 \mathrm{E}-11$ & $3.130 \mathrm{E}+24$ & $4.382 \mathrm{E}+24$ & $5.239 \mathrm{E}+19$ & $4.070 \mathrm{E}+19$ \\
\hline $2.269 \mathrm{E}+22$ & $4.61 \mathrm{E}+20$ & $8.16 \mathrm{E}-21$ & $2.10 \mathrm{E}+09$ & $9.804 \mathrm{E}-12$ & $1.429 \mathrm{E}+25$ & $2.031 \mathrm{E}+25$ & $1.695 \mathrm{E}+20$ & $1.302 \mathrm{E}+20$ \\
\hline $2.269 \mathrm{E}+23$ & $1.49 \mathrm{E}+21$ & $8.37 \mathrm{E}-22$ & $2.05 \mathrm{E}+09$ & $9.839 \mathrm{E}-13$ & $6.523 \mathrm{E}+25$ & $9.424 \mathrm{E}+25$ & $5.491 \mathrm{E}+20$ & $4.167 \mathrm{E}+20$ \\
\hline $2.269 \mathrm{E}+24$ & $4.84 \mathrm{E}+21$ & 8.57E-23 & $2.00 \mathrm{E}+09$ & $9.859 \mathrm{E}-14$ & $2.980 \mathrm{E}+26$ & $4.378 \mathrm{E}+26$ & $1.781 \mathrm{E}+21$ & $1.334 \mathrm{E}+21$ \\
\hline $2.269 \mathrm{E}+25$ & $1.57 \mathrm{E}+22$ & $8.77 \mathrm{E}-24$ & $1.95 \mathrm{E}+09$ & $9.863 \mathrm{E}-15$ & $1.362 \mathrm{E}+27$ & $2.036 \mathrm{E}+27$ & $5.782 \mathrm{E}+21$ & $4.276 \mathrm{E}+21$ \\
\hline $2.269 \mathrm{E}+26$ & $5.12 \mathrm{E}+22$ & $8.98 \mathrm{E}-25$ & $1.89 \mathrm{E}+09$ & $9.849 \mathrm{E}-16$ & $6.225 \mathrm{E}+27$ & $9.483 \mathrm{E}+27$ & $1.881 \mathrm{E}+22$ & $1.371 \mathrm{E}+22$ \\
\hline $2.269 \mathrm{E}+27$ & $1.67 \mathrm{E}+23$ & $9.18 \mathrm{E}-26$ & $1.84 \mathrm{E}+09$ & $9.817 \mathrm{E}-17$ & $2.846 \mathrm{E}+28$ & $4.423 \mathrm{E}+28$ & $6.126 \mathrm{E}+22$ & $4.401 \mathrm{E}+22$ \\
\hline $2.269 \mathrm{E}+28$ & $5.44 \mathrm{E}+23$ & $9.38 \mathrm{E}-27$ & $1.78 \mathrm{E}+09$ & $9.766 \mathrm{E}-18$ & $1.302 \mathrm{E}+29$ & $2.066 \mathrm{E}+29$ & $1.999 \mathrm{E}+23$ & $1.414 \mathrm{E}+23$ \\
\hline $2.269 \mathrm{E}+29$ & $1.78 \mathrm{E}+24$ & $9.59 \mathrm{E}-28$ & $1.72 \mathrm{E}+09$ & $9.695 \mathrm{E}-19$ & $5.958 \mathrm{E}+29$ & $9.670 \mathrm{E}+29$ & $6.539 \mathrm{E}+23$ & $4.547 \mathrm{E}+23$ \\
\hline $2.269 \mathrm{E}+30$ & $5.83 \mathrm{E}+24$ & $9.79 \mathrm{E}-29$ & $1.66 \mathrm{E}+09$ & $9.601 \mathrm{E}-20$ & $2.727 \mathrm{E}+30$ & $4.534 \mathrm{E}+30$ & $2.144 \mathrm{E}+24$ & $1.464 \mathrm{E}+24$ \\
\hline $2.269 \mathrm{E}+31$ & $1.92 \mathrm{E}+25$ & $9.99 \mathrm{E}-30$ & $1.60 \mathrm{E}+09$ & $9.483 \mathrm{E}-21$ & $1.249 \mathrm{E}+31$ & $2.131 \mathrm{E}+31$ & $7.049 \mathrm{E}+24$ & $4.721 \mathrm{E}+24$ \\
\hline $2.269 \mathrm{E}+32$ & $6.33 \mathrm{E}+25$ & $1.02 \mathrm{E}-30$ & $1.53 \mathrm{E}+09$ & $9.339 \mathrm{E}-22$ & $5.718 \mathrm{E}+31$ & $1.004 \mathrm{E}+32$ & $2.325 \mathrm{E}+25$ & $1.525 \mathrm{E}+25$ \\
\hline $2.269 \mathrm{E}+33$ & $2.09 \mathrm{E}+26$ & $1.04 \mathrm{E}-31$ & $1.46 \mathrm{E}+09$ & $9.166 \mathrm{E}-23$ & $2.620 \mathrm{E}+32$ & $4.741 \mathrm{E}+32$ & $7.700 \mathrm{E}+25$ & $4.935 \mathrm{E}+25$ \\
\hline $2.269 \mathrm{E}+34$ & $6.97 \mathrm{E}+26$ & $1.06 \mathrm{E}-32$ & $1.39 \mathrm{E}+09$ & $8.960 \mathrm{E}-24$ & $1.200 \mathrm{E}+33$ & $2.247 \mathrm{E}+33$ & $2.562 \mathrm{E}+26$ & $1.601 \mathrm{E}+26$ \\
\hline $2.269 \mathrm{E}+35$ & $2.33 \mathrm{E}+27$ & $1.08 \mathrm{E}-33$ & $1.31 \mathrm{E}+09$ & $8.719 \mathrm{E}-25$ & $5.501 \mathrm{E}+33$ & $1.070 \mathrm{E}+34$ & $8.573 \mathrm{E}+26$ & $5.207 \mathrm{E}+26$ \\
\hline $2.269 \mathrm{E}+36$ & $7.86 \mathrm{E}+27$ & $1.10 \mathrm{E}-34$ & $1.23 \mathrm{E}+09$ & $8.435 \mathrm{E}-26$ & $2.522 \mathrm{E}+34$ & $5.117 \mathrm{E}+34$ & $2.890 \mathrm{E}+27$ & $1.700 \mathrm{E}+27$ \\
\hline $2.269 \mathrm{E}+37$ & $2.67 \mathrm{E}+28$ & $1.12 \mathrm{E}-35$ & $1.15 \mathrm{E}+09$ & $8.103 \mathrm{E}-27$ & $1.156 \mathrm{E}+35$ & $2.463 \mathrm{E}+35$ & $9.831 \mathrm{E}+27$ & $5.576 \mathrm{E}+27$ \\
\hline $2.269 \mathrm{E}+38$ & $9.22 \mathrm{E}+28$ & $1.14 \mathrm{E}-36$ & $1.05 \mathrm{E}+09$ & $7.713 \mathrm{E}-28$ & $5.304 \mathrm{E}+35$ & $1.196 \mathrm{E}+36$ & $3.387 \mathrm{E}+28$ & $1.840 \mathrm{E}+28$ \\
\hline $2.269 \mathrm{E}+39$ & $3.23 \mathrm{E}+29$ & $1.16 \mathrm{E}-37$ & $9.48 \mathrm{E}+08$ & $7.249 \mathrm{E}-29$ & $2.433 \mathrm{E}+36$ & $5.880 \mathrm{E}+36$ & $1.188 \mathrm{E}+29$ & $6.129 \mathrm{E}+28$ \\
\hline $2.269 \mathrm{E}+40$ & $1.16 \mathrm{E}+30$ & $1.18 \mathrm{E}-38$ & $8.32 \mathrm{E}+08$ & $6.689 \mathrm{E}-30$ & $1.116 \mathrm{E}+37$ & $2.943 \mathrm{E}+37$ & $4.281 \mathrm{E}+29$ & $2.069 \mathrm{E}+29$ \\
\hline $2.269 \mathrm{E}+41$ & $4.40 \mathrm{E}+30$ & $1.20 \mathrm{E}-39$ & $6.97 \mathrm{E}+08$ & $5.991 \mathrm{E}-31$ & $5.123 \mathrm{E}+37$ & $1.520 \mathrm{E}+38$ & $1.617 \mathrm{E}+30$ & $7.150 \mathrm{E}+29$ \\
\hline $2.269 \mathrm{E}+42$ & $1.84 \mathrm{E}+31$ & $1.22 \mathrm{E}-40$ & $5.28 \mathrm{E}+08$ & $5.059 \mathrm{E}-32$ & $2.352 \mathrm{E}+38$ & $8.398 \mathrm{E}+38$ & $6.749 \mathrm{E}+30$ & $2.598 \mathrm{E}+30$ \\
\hline
\end{tabular}

\section{Application-5: To estimate the current distance travelled by the universe}

According to modern cosmological observations, the commoving distance from Earth to the edge of the observable universe is about $14.26 \mathrm{Gpc}\left(46.5 \mathrm{Gly}=4.40 \times 10^{26}\right.$ meters $)$ in any direction. The observable universe is thus a sphere with a diameter of about $28.5 \mathrm{Gpc}=93$ Gly $=8.8 \times 10^{26} \mathrm{~m}$ ). Readers are suggested to see the valuable scientific information available in Wikipedia web site on 'Observational cosmology'.

According to Mihran Vardanyan et al [25], "Bayesian model averaging is a procedure to obtain parameter constraints that account for the uncertainty about the correct cosmological model. We use recent cosmological observations and Bayesian model averaging to derive tight limits on the curvature parameter, as well as robust lower bounds on the curvature radius of the Universe and its minimum size, while allowing for the possibility of an evolving dark energy component. Because flat models are favored by Bayesian model selection, we find that model-averaged constraints on the curvature and size of the Universe can be 
considerably stronger than non model-averaged ones. For the most conservative prior choice (based on inflationary considerations), our procedure improves on non model- averaged constraints on the curvature by a factor of 2 . The curvature scale of the Universe is conservatively constrained to be $R_{c}>42 \mathrm{Gpc}(99 \%)$, corresponding to a lower limit to the number of Hubble spheres in the Universe NU > 251 (99\%)".

With reference to our proposed assumptions, current cosmic radius (including observable and non-observable) can be estimated in the following way. From the beginning of Planck scale which is assumed to be associated with big bang, cosmic distance travelled can be estimated as follows:

$$
S_{t} \cong R_{t} \cong\left(\frac{V_{t}+c}{2}\right) t_{t} \cong\left(\frac{V_{t}+c}{2}\right)\left\{\left[1+\ln \left(\frac{H_{t}}{H_{0}}\right)^{2}\right]\left(\frac{1}{H_{0}}\right)\right\}
$$

For the current case,

$$
\begin{aligned}
S_{0} & \cong R_{0} \cong\left(\frac{V_{0}+c}{2}\right) t_{0} \cong\left(\frac{c}{2 H_{0}}\right)\left[1+\sqrt{1+\ln \left(\frac{H_{p l}}{H_{0}}\right)}\right] \\
& \cong 6.4425\left(\frac{c}{H_{0}}\right) \cong 8.514 \times 10^{26} \mathrm{~m}
\end{aligned}
$$

From our estimate, current distance (observable and non-observable) about the point of big bang is $90 \mathrm{Gly}=27.6 \mathrm{Gpc}$. See the following table-3. Clearly speaking, current universe seems to constitute 267 Hubble spheres [25].This is really a very interesting coincidence and needs further study at fundamental level. Our estimate seems to be approximately 1.935 times higher than modern estimation. With further research and analysis and by understanding the galactic red shifts, discrepancy can be reviewed and resolved. Diameter of current (observable and non-observable) cosmic sphere about the point of big bang is 180 Gly=55.2 Gpc. See the following tables 3 and 4.

Table-3: To fit the current cosmic distance

\begin{tabular}{|l|c|l|}
\hline $\begin{array}{l}\text { Estimating } \\
\text { method }\end{array}$ & $\begin{array}{l}\text { Cosmic } \\
\text { distance }\end{array}$ & Reference point \\
\hline $\begin{array}{l}\text { Modern estimate } \\
\text { (only observable) }\end{array}$ & $\begin{array}{c}46.5 \mathrm{Gly} \\
14.26 \mathrm{Gpc}\end{array}$ & About earth \\
\hline $\begin{array}{c}\text { Our estimate } \\
\text { (Observable }+\end{array}$ & $\begin{array}{l}90.0 \mathrm{Gly} \\
27.6 \mathrm{Gpc}\end{array}$ & $\begin{array}{l}\text { About point of } \\
\text { big bang }\end{array}$ \\
non-observable) & Note: Gly $=$ Giga or billion light years \\
Gpc $=$ Giga or billion parsec \\
\hline
\end{tabular}


Table-4: To fit the current cosmic diameter

\begin{tabular}{|l|c|l|}
\hline $\begin{array}{l}\text { Estimating } \\
\text { method }\end{array}$ & $\begin{array}{l}\text { Cosmic } \\
\text { diameter }\end{array}$ & Reference point \\
\hline $\begin{array}{c}\text { Modern estimate } \\
\text { (only observable) }\end{array}$ & $\begin{array}{c}93 \mathrm{Gly} \\
28.5 \mathrm{Gpc}\end{array}$ & About earth \\
\hline $\begin{array}{c}\text { Our estimate } \\
\text { (Observable }+ \\
\text { non-observable) }\end{array}$ & $\begin{array}{c}180 \mathrm{Gly} \\
55.2 \mathrm{Gpc}\end{array}$ & $\begin{array}{c}\text { About point of } \\
\text { big bang }\end{array}$ \\
\hline
\end{tabular}

\section{Application-6: To estimate the galactic receding speeds and galactic distances in the current accelerating universe}

Based on relations (20) and (21), within the current radius of $90 \mathrm{Gly}=27.6 \mathrm{Gpc}$, from and about the point of big bang, galactic receding speeds can be approximated with the following relation.

$$
v_{g} \cong\left(\frac{d_{g}}{S_{0}}\right) V_{0} \cong\left(\frac{d_{g}}{S_{0}}\right) 11.8851 c \cong\left\{\frac{2 \sqrt{1+\ln \left(H_{p l} / H_{0}\right)}}{\left[1+\sqrt{1+\ln \left(H_{p l} / H_{0}\right)}\right]}\right\}\left(d_{g} H_{0}\right)
$$

where $d_{g}$ is the current galactic distance from the point of big bang and $v_{g}$ is the current galactic receding speed.

Based on this relation (22), within the current boundary of $90 \mathrm{Gly}=27.6 \mathrm{Gpc}$, galactic distances corresponding to assumed galactic receding speeds can be expressed in the following way.

$$
d_{g} \cong\left(\frac{v_{g}}{V_{0}}\right) S_{0} \cong\left(\frac{v_{g}}{11.8851 c}\right) 8.514 \times 10^{26} \mathrm{~m}
$$

See the following table-5. By co-relating the estimated galactic distances and actual galactic receding speeds with observed galactic red shifts, further research can be carried out.

Table-5: To understand galactic receding speeds and distances from and about the point of big bang

\begin{tabular}{|c|c|c|c|}
\hline \multirow{2}{*}{$\begin{array}{c}\text { Galactic } \\
\text { receding } \\
\text { speed }\end{array}$} & \multicolumn{3}{|c|}{$\begin{array}{c}\text { Estimated distance from and about } \\
\text { the point of big bang }\end{array}$} \\
\cline { 2 - 4 } & meters & Gly & Gpc \\
\hline $0.1 c$ & $7.164 \mathrm{E}+24$ & 0.76 & 0.23 \\
\hline $0.2 c$ & $1.433 \mathrm{E}+25$ & 1.51 & 0.46 \\
\hline $0.3 c$ & $2.149 \mathrm{E}+25$ & 2.27 & 0.70 \\
\hline $0.4 c$ & $2.865 \mathrm{E}+25$ & 3.03 & 0.93 \\
\hline $0.5 c$ & $3.582 \mathrm{E}+25$ & 3.79 & 1.16 \\
\hline $0.6 c$ & $4.298 \mathrm{E}+25$ & 4.54 & 1.39 \\
\hline
\end{tabular}




\begin{tabular}{|c|c|c|c|}
\hline $0.7 c$ & $5.015 \mathrm{E}+25$ & 5.30 & 1.63 \\
\hline $0.8 c$ & $5.731 \mathrm{E}+25$ & 6.06 & 1.86 \\
\hline $0.9 c$ & $6.447 \mathrm{E}+25$ & 6.81 & 2.09 \\
\hline$c$ & $7.164 \mathrm{E}+25$ & 7.57 & 2.32 \\
\hline $1.1 c$ & $7.880 \mathrm{E}+25$ & 8.33 & 2.55 \\
\hline $1.2 c$ & $8.596 \mathrm{E}+25$ & 9.09 & 2.79 \\
\hline $1.3 c$ & $9.313 \mathrm{E}+25$ & 9.84 & 3.02 \\
\hline $1.4 c$ & $1.003 \mathrm{E}+26$ & 10.60 & 3.25 \\
\hline $1.5 c$ & $1.075 \mathrm{E}+26$ & 11.36 & 3.48 \\
\hline $1.6 c$ & $1.146 \mathrm{E}+26$ & 12.12 & 3.72 \\
\hline $1.7 c$ & $1.218 \mathrm{E}+26$ & 12.87 & 3.95 \\
\hline $1.8 c$ & $1.289 \mathrm{E}+26$ & 13.63 & 4.18 \\
\hline $1.9 c$ & $1.361 \mathrm{E}+26$ & 14.39 & 4.41 \\
\hline $2 c$ & $1.433 \mathrm{E}+26$ & 15.14 & 4.65 \\
\hline
\end{tabular}

\section{Application-7: To estimate the current mass and mass density of the universe}

It may be noted that, many scientists are thinking on 'understanding the mass of universe' in terms of 'Mach's principle' i.e "relation between inertia and gravity"[17-24]. In this context, our proposed assumption, $3 \mathrm{~b}$ can be given some consideration at fundamantal level. One can find interesting trechnical discussion on this assumption by D.W.Sciama, R.H. Dicke, C. Brans, G. J. Whitrow, F. M. Gomide, Berman M.S, Som.M.M and A.I. Arbab.

With reference to assumption $3 \mathrm{~b}$, at any stage of cosmic evolution,

$$
M_{t} \cong \frac{c^{2} R_{t}}{G}
$$

From applications 2 and 3, current visual and non-visual cosmic radius is around 90 Gly. With reference to assumption $3 \mathrm{~b}$, current mass of our (visible and invisible parts) universe can be estimated with the following relation.

$$
M_{0} \cong \frac{c^{2} R_{0}}{G} \cong 1.14654 \times 10^{54} \mathrm{~kg}
$$

where, $R_{0} \cong\left(\frac{V_{0}+c}{2}\right)\left(\frac{1}{H_{0}}\right)$. It may be noted that, with this mass, current mass energy density can be expressed with the following relation. 


$$
\begin{aligned}
& \left(\rho_{\text {mass }}\right)_{0} \cong \frac{M_{0}}{(4 \pi / 3) R_{0}^{3}} \cong \frac{3 c^{2}}{4 \pi G R_{0}^{2}} \\
& \cong\left[1+\sqrt{1+\ln \left(\frac{H_{p l}}{H_{0}}\right)}\right]^{-2} \frac{3 H_{0}^{2}}{\pi G} \cong 4.43505 \times 10^{-28} \mathrm{~kg} \cdot \mathrm{m}^{-3}
\end{aligned}
$$

Now mass energy density can be expressed with the following relation.

$$
\begin{aligned}
& \left(\rho_{\text {mass }} c^{2}\right)_{0} \cong \frac{M_{0} c^{2}}{(4 \pi / 3) R_{0}^{3}} \cong \frac{3 c^{4}}{4 \pi G R_{0}^{2}} \\
& \cong\left[1+\sqrt{1+\ln \left(\frac{H_{p l}}{H_{0}}\right)}\right]^{-2} \frac{3 H_{0}^{2} c^{2}}{\pi G} \cong 3.9865 \times 10^{-11} \mathrm{~J} . \mathrm{m}^{-3}
\end{aligned}
$$

Now ratio of mass energy density and critical energy density [14] can be expressed with the following relation.

$$
\left(\frac{\rho_{\text {mass }} c^{2}}{\rho_{\text {critical }} c^{2}}\right)_{0} \cong 8\left[1+\sqrt{1+\ln \left(\frac{H_{p l}}{H_{0}}\right)}\right]^{-2} \cong 0.048185
$$

Based on this strange and interesting coincidence, proposed method of estimating the current cosmic mass can be given some consideration. Clearly speaking, Mach's principle and its quantitative relation, $G M_{0} \cong c^{2} R_{0}$ seems to play a vital role in understanding the large scale structure of the evolving universe and its mass density. It may be noted that, in our earlier published papers and references there in, we have focused on $\frac{G M_{0}}{c^{2} R_{0}} \cong \frac{1}{2}$ and developed "Black hole cosmology models". But we could not present a reasonable explanation for the origin and understanding of the observed cosmic visible mass density. See the following table- 6 for understanding the past cosmic mass density. From the last column of table- 6 , it is clear that, during cosmic evolution, mass density increases with cosmic age and strongly supports the 'hot big bang' idea of 'early matter was in the form of radiation'.

Table-6: To understand the past cosmic mass density

\begin{tabular}{|c|c|c|c|c|c|c|c|}
\hline $\begin{array}{c}\text { Assumed } \\
\text { Hubble } \\
\text { parameter } \\
(1 / \mathrm{sec})\end{array}$ & $\begin{array}{c}\text { Estimated } \\
\text { Cosmic } \\
\text { age } \\
(\mathrm{sec})\end{array}$ & $\begin{array}{c}\text { Estimated } \\
\text { cosmic } \\
\text { radius } \\
(\mathrm{m})\end{array}$ & $\begin{array}{c}\text { Estimated } \\
\text { cosmic } \\
\text { mass } \\
(\mathrm{kg})\end{array}$ & $\begin{array}{c}\text { Estimated } \\
\text { cosmic } \\
\text { volume } \\
\left(\mathrm{m}^{3}\right)\end{array}$ & $\begin{array}{c}\text { Estimated } \\
\text { cosmic } \\
\text { mass } \\
\text { density } \\
\left(\mathrm{kg} / \mathrm{m}^{3}\right)\end{array}$ & $\begin{array}{c}\text { Estimated } \\
\text { critical } \\
\text { density } \\
\left(\mathrm{kg} / \mathrm{m}^{3}\right.\end{array}$ & $\begin{array}{c}\text { Ratio of mass } \\
\text { density and } \\
\text { critical density }\end{array}$ \\
\hline $2.269 \mathrm{E}-18$ & $4.41 \mathrm{E}+17$ & $8.514 \mathrm{E}+26$ & $1.147 \mathrm{E}+54$ & $2.585 \mathrm{E}+81$ & $4.435 \mathrm{E}-28$ & $9.204 \mathrm{E}-27$ & $4.819 \mathrm{E}-02$ \\
\hline $2.269 \mathrm{E}-17$ & $2.47 \mathrm{E}+17$ & $4.736 \mathrm{E}+26$ & $6.378 \mathrm{E}+53$ & $4.450 \mathrm{E}+80$ & $1.433 \mathrm{E}-27$ & $9.204 \mathrm{E}-25$ & $1.557 \mathrm{E}-03$ \\
\hline $2.269 \mathrm{E}-16$ & $4.50 \mathrm{E}+16$ & $8.561 \mathrm{E}+25$ & $1.153 \mathrm{E}+53$ & $2.629 \mathrm{E}+78$ & $4.386 \mathrm{E}-26$ & $9.204 \mathrm{E}-23$ & $4.765 \mathrm{E}-04$ \\
\hline $2.269 \mathrm{E}-15$ & $6.53 \mathrm{E}+15$ & $1.233 \mathrm{E}+25$ & $1.660 \mathrm{E}+52$ & $7.844 \mathrm{E}+75$ & $2.116 \mathrm{E}-24$ & $9.204 \mathrm{E}-21$ & $2.299 \mathrm{E}-04$ \\
\hline
\end{tabular}




\begin{tabular}{|c|c|c|c|c|c|c|c|}
\hline $2.269 \mathrm{E}-14$ & $8.56 \mathrm{E}+14$ & $1.603 \mathrm{E}+24$ & $2.159 \mathrm{E}+51$ & $1.725 \mathrm{E}+73$ & $1.251 \mathrm{E}-22$ & 9.204E-19 & $1.359 \mathrm{E}-04$ \\
\hline $2.269 \mathrm{E}-13$ & $1.06 \mathrm{E}+14$ & $1.967 \mathrm{E}+23$ & $2.649 \mathrm{E}+50$ & $3.188 \mathrm{E}+70$ & 8.309E-21 & $9.204 \mathrm{E}-17$ & $9.027 \mathrm{E}-05$ \\
\hline 2.473E-12 & $1.16 \mathrm{E}+13$ & $2.145 E+22$ & $2.888 \mathrm{E}+49$ & $4.132 \mathrm{E}+67$ & 6.990E-19 & 1.094E-14 & $6.389 E-05$ \\
\hline $2.269 \mathrm{E}-12$ & $1.26 \mathrm{E}+13$ & $2.325 \mathrm{E}+22$ & $3.131 \mathrm{E}+49$ & $5.264 \mathrm{E}+67$ & 5.948E-19 & $9.204 \mathrm{E}-15$ & $6.462 \mathrm{E}-05$ \\
\hline $2.269 \mathrm{E}-11$ & $1.47 \mathrm{E}+12$ & $2.676 \mathrm{E}+21$ & $3.604 \mathrm{E}+48$ & $8.030 \mathrm{E}+64$ & $4.488 \mathrm{E}-17$ & $9.204 \mathrm{E}-13$ & $4.876 \mathrm{E}-05$ \\
\hline $2.269 \mathrm{E}-10$ & $1.67 \mathrm{E}+11$ & $3.021 \mathrm{E}+20$ & $4.069 \mathrm{E}+47$ & $1.155 \mathrm{E}+62$ & $3.522 \mathrm{E}-15$ & $9.204 \mathrm{E}-11$ & $3.826 \mathrm{E}-05$ \\
\hline 2.269E-09 & $1.87 \mathrm{E}+10$ & $3.360 \mathrm{E}+19$ & $4.524 \mathrm{E}+46$ & $1.589 \mathrm{E}+59$ & $2.848 \mathrm{E}-13$ & 9.204E-09 & 3.094E-05 \\
\hline $2.269 \mathrm{E}-08$ & $2.07 \mathrm{E}+09$ & $3.691 \mathrm{E}+18$ & $4.971 \mathrm{E}+45$ & $2.107 \mathrm{E}+56$ & $2.359 \mathrm{E}-11$ & 9.204E-07 & $2.563 \mathrm{E}-05$ \\
\hline $2.269 \mathrm{E}-07$ & $2.28 \mathrm{E}+08$ & $4.016 \mathrm{E}+17$ & $5.409 \mathrm{E}+44$ & $2.714 \mathrm{E}+53$ & 1.993E-09 & $9.204 \mathrm{E}-05$ & $2.165 \mathrm{E}-05$ \\
\hline $2.269 \mathrm{E}-06$ & $2.48 \mathrm{E}+07$ & $4.335 \mathrm{E}+16$ & $5.837 \mathrm{E}+43$ & $3.411 \mathrm{E}+50$ & $1.711 \mathrm{E}-07$ & 9.204E-03 & $1.859 \mathrm{E}-05$ \\
\hline $2.269 \mathrm{E}-05$ & $2.68 \mathrm{E}+06$ & $4.646 \mathrm{E}+15$ & $6.256 \mathrm{E}+42$ & $4.200 \mathrm{E}+47$ & $1.490 \mathrm{E}-05$ & $9.204 \mathrm{E}-01$ & $1.618 \mathrm{E}-05$ \\
\hline $2.269 \mathrm{E}-04$ & $2.89 \mathrm{E}+05$ & $4.950 \mathrm{E}+14$ & $6.665 \mathrm{E}+41$ & $5.080 \mathrm{E}+44$ & $1.312 \mathrm{E}-03$ & $9.204 \mathrm{E}+01$ & $1.426 \mathrm{E}-05$ \\
\hline $2.269 \mathrm{E}-03$ & $3.09 \mathrm{E}+04$ & $5.247 \mathrm{E}+13$ & $7.065 \mathrm{E}+40$ & $6.049 \mathrm{E}+41$ & $1.168 \mathrm{E}-01$ & $9.204 \mathrm{E}+03$ & $1.269 \mathrm{E}-05$ \\
\hline 2.269E-02 & $3.29 \mathrm{E}+03$ & $5.536 \mathrm{E}+12$ & $7.455 \mathrm{E}+39$ & $7.107 \mathrm{E}+38$ & $1.049 \mathrm{E}+01$ & $9.204 \mathrm{E}+05$ & $1.140 \mathrm{E}-05$ \\
\hline $2.269 \mathrm{E}-01$ & $3.50 \mathrm{E}+02$ & $5.818 \mathrm{E}+11$ & $7.835 \mathrm{E}+38$ & $8.249 \mathrm{E}+35$ & $9.498 \mathrm{E}+02$ & $9.204 \mathrm{E}+07$ & $1.032 \mathrm{E}-05$ \\
\hline $2.269 \mathrm{E}+00$ & $3.70 \mathrm{E}+01$ & $6.092 \mathrm{E}+10$ & $8.204 \mathrm{E}+37$ & $9.472 \mathrm{E}+32$ & $8.661 \mathrm{E}+04$ & $9.204 \mathrm{E}+09$ & $9.410 \mathrm{E}-06$ \\
\hline $2.269 \mathrm{E}+01$ & $3.90 \mathrm{E}+00$ & $6.359 \mathrm{E}+09$ & $8.563 \mathrm{E}+36$ & $1.077 \mathrm{E}+30$ & $7.950 \mathrm{E}+06$ & $9.204 \mathrm{E}+11$ & 8.638E-06 \\
\hline $2.269 \mathrm{E}+02$ & $4.10 \mathrm{E}-01$ & $6.618 \mathrm{E}+08$ & $8.912 \mathrm{E}+35$ & $1.214 \mathrm{E}+27$ & $7.341 \mathrm{E}+08$ & $9.204 \mathrm{E}+13$ & $7.975 \mathrm{E}-06$ \\
\hline $2.269 \mathrm{E}+03$ & $4.31 \mathrm{E}-02$ & $6.869 \mathrm{E}+07$ & $9.249 E+34$ & $1.357 \mathrm{E}+24$ & $6.815 \mathrm{E}+10$ & $9.204 \mathrm{E}+15$ & 7.404E-06 \\
\hline $2.269 \mathrm{E}+04$ & $4.51 \mathrm{E}-03$ & $7.111 \mathrm{E}+06$ & $9.576 \mathrm{E}+33$ & $1.506 \mathrm{E}+21$ & $6.358 \mathrm{E}+12$ & $9.204 \mathrm{E}+17$ & $6.908 \mathrm{E}-06$ \\
\hline $2.269 \mathrm{E}+05$ & $4.71 \mathrm{E}-04$ & $7.345 \mathrm{E}+05$ & $9.891 \mathrm{E}+32$ & $1.660 \mathrm{E}+18$ & $5.959 \mathrm{E}+14$ & $9.204 \mathrm{E}+19$ & $6.474 \mathrm{E}-06$ \\
\hline $2.269 \mathrm{E}+06$ & $4.92 \mathrm{E}-05$ & $7.571 \mathrm{E}+04$ & $1.019 \mathrm{E}+32$ & $1.817 \mathrm{E}+15$ & $5.609 \mathrm{E}+16$ & $9.204 \mathrm{E}+21$ & $6.094 \mathrm{E}-06$ \\
\hline $2.269 \mathrm{E}+07$ & $5.12 \mathrm{E}-06$ & $7.787 \mathrm{E}+03$ & $1.049 \mathrm{E}+31$ & $1.978 \mathrm{E}+12$ & $5.301 \mathrm{E}+18$ & $9.204 \mathrm{E}+23$ & $5.760 \mathrm{E}-06$ \\
\hline $2.269 \mathrm{E}+08$ & $5.32 \mathrm{E}-07$ & $7.995 \mathrm{E}+02$ & $1.077 \mathrm{E}+30$ & $2.141 \mathrm{E}+09$ & $5.030 \mathrm{E}+20$ & $9.204 \mathrm{E}+25$ & $5.465 \mathrm{E}-06$ \\
\hline $2.269 \mathrm{E}+09$ & $5.53 \mathrm{E}-08$ & $8.193 \mathrm{E}+01$ & $1.103 \mathrm{E}+29$ & $2.304 \mathrm{E}+06$ & $4.789 \mathrm{E}+22$ & $9.204 \mathrm{E}+27$ & $5.203 \mathrm{E}-06$ \\
\hline $2.269 \mathrm{E}+10$ & 5.73E-09 & $8.382 \mathrm{E}+00$ & $1.129 \mathrm{E}+28$ & $2.467 \mathrm{E}+03$ & $4.575 \mathrm{E}+24$ & $9.204 \mathrm{E}+29$ & $4.971 \mathrm{E}-06$ \\
\hline $2.269 \mathrm{E}+11$ & $5.93 \mathrm{E}-10$ & $8.562 \mathrm{E}-01$ & $1.153 \mathrm{E}+27$ & $2.629 \mathrm{E}+00$ & $4.386 \mathrm{E}+26$ & $9.204 \mathrm{E}+31$ & 4.765E-06 \\
\hline $2.269 \mathrm{E}+12$ & $6.13 \mathrm{E}-11$ & $8.731 \mathrm{E}-02$ & $1.176 \mathrm{E}+26$ & $2.788 \mathrm{E}-03$ & $4.217 \mathrm{E}+28$ & $9.204 \mathrm{E}+33$ & $4.582 \mathrm{E}-06$ \\
\hline $2.269 \mathrm{E}+13$ & 6.34 & $8.890 \mathrm{E}-03$ & $1.197 \mathrm{E}+25$ & $2.943 \mathrm{E}-06$ & $4.067 \mathrm{E}+30$ & $9.204 \mathrm{E}+35$ & 4.419E-06 \\
\hline $2.269 \mathrm{E}+14$ & $6.54 \mathrm{E}-13$ & $9.039 \mathrm{E}-04$ & $1.217 \mathrm{E}+24$ & 3.094E-09 & $3.935 \mathrm{E}+32$ & $9.204 \mathrm{E}+37$ & $4.275 \mathrm{E}-06$ \\
\hline $2.269 \mathrm{E}+15$ & $6.74 \mathrm{E}-14$ & $9.177 \mathrm{E}-05$ & $1.236 \mathrm{E}+23$ & $3.237 \mathrm{E}-12$ & $3.817 \mathrm{E}+34$ & $9.204 \mathrm{E}+39$ & $4.148 \mathrm{E}-06$ \\
\hline $2.269 \mathrm{E}+16$ & $6.95 \mathrm{E}-15$ & $9.303 \mathrm{E}-06$ & $1.253 \mathrm{E}+22$ & $3.373 \mathrm{E}-15$ & $3.714 \mathrm{E}+36$ & $9.204 \mathrm{E}+41$ & $4.035 \mathrm{E}-06$ \\
\hline $2.269 \mathrm{E}+17$ & $7.15 \mathrm{E}-16$ & $9.418 \mathrm{E}-07$ & $1.268 \mathrm{E}+21$ & $3.500 \mathrm{E}-18$ & $3.624 \mathrm{E}+38$ & $9.204 \mathrm{E}+43$ & $3.938 \mathrm{E}-06$ \\
\hline $2.269 \mathrm{E}+18$ & $7.35 \mathrm{E}-17$ & $9.521 \mathrm{E}-08$ & $1.282 \mathrm{E}+20$ & $3.616 \mathrm{E}-21$ & $3.546 \mathrm{E}+40$ & $9.204 \mathrm{E}+45$ & $3.853 \mathrm{E}-06$ \\
\hline $2.269 \mathrm{E}+19$ & $7.56 \mathrm{E}-18$ & $9.612 \mathrm{E}-09$ & $1.294 \mathrm{E}+19$ & $3.720 \mathrm{E}-24$ & $3.480 \mathrm{E}+42$ & $9.204 \mathrm{E}+47$ & $3.781 \mathrm{E}-06$ \\
\hline $2.269 \mathrm{E}+20$ & $7.76 \mathrm{E}-19$ & $9.689 \mathrm{E}-10$ & $1.305 \mathrm{E}+18$ & $3.811 \mathrm{E}-27$ & $3.424 \mathrm{E}+44$ & $9.204 \mathrm{E}+49$ & $3.720 \mathrm{E}-06$ \\
\hline $2.269 \mathrm{E}+21$ & $7.96 \mathrm{E}-20$ & $9.754 \mathrm{E}-11$ & $1.313 \mathrm{E}+17$ & $3.887 \mathrm{E}-30$ & $3.379 \mathrm{E}+46$ & $9.204 \mathrm{E}+51$ & $3.672 \mathrm{E}-06$ \\
\hline $2.269 \mathrm{E}+22$ & $8.16 \mathrm{E}-21$ & $9.804 \mathrm{E}-12$ & $1.320 \mathrm{E}+16$ & $3.947 \mathrm{E}-33$ & $3.345 \mathrm{E}+48$ & $9.204 \mathrm{E}+53$ & $3.634 \mathrm{E}-06$ \\
\hline $2.269 \mathrm{E}+23$ & $8.37 \mathrm{E}-22$ & $9.839 \mathrm{E}-13$ & $1.325 \mathrm{E}+15$ & $3.990 \mathrm{E}-36$ & $3.321 \mathrm{E}+50$ & $9.204 \mathrm{E}+55$ & $3.608 \mathrm{E}-06$ \\
\hline $2.269 \mathrm{E}+24$ & $8.57 \mathrm{E}-23$ & $9.859 \mathrm{E}-14$ & $1.328 \mathrm{E}+14$ & 4.014E-39 & $3.308 \mathrm{E}+52$ & $9.204 \mathrm{E}+57$ & $3.594 \mathrm{E}-06$ \\
\hline $2.269 \mathrm{E}+25$ & $8.77 \mathrm{E}-24$ & $9.863 \mathrm{E}-15$ & $1.328 \mathrm{E}+13$ & $4.018 \mathrm{E}-42$ & $3.305 \mathrm{E}+54$ & $9.204 \mathrm{E}+59$ & $3.591 \mathrm{E}-06$ \\
\hline
\end{tabular}




\begin{tabular}{|c|c|c|c|c|c|c|c|}
\hline $2.269 \mathrm{E}+26$ & $8.98 \mathrm{E}-25$ & $9.849 \mathrm{E}-16$ & $1.326 \mathrm{E}+12$ & $4.002 \mathrm{E}-45$ & $3.314 \mathrm{E}+56$ & $9.204 \mathrm{E}+61$ & $3.601 \mathrm{E}-06$ \\
\hline $2.269 \mathrm{E}+27$ & $9.18 \mathrm{E}-26$ & $9.817 \mathrm{E}-17$ & $1.322 \mathrm{E}+11$ & $3.964 \mathrm{E}-48$ & $3.336 \mathrm{E}+58$ & $9.204 \mathrm{E}+63$ & $3.624 \mathrm{E}-06$ \\
\hline $2.269 \mathrm{E}+28$ & $9.38 \mathrm{E}-27$ & $9.766 \mathrm{E}-18$ & $1.315 \mathrm{E}+10$ & $3.902 \mathrm{E}-51$ & $3.370 \mathrm{E}+60$ & $9.204 \mathrm{E}+65$ & $3.662 \mathrm{E}-06$ \\
\hline $2.269 \mathrm{E}+29$ & $9.59 \mathrm{E}-28$ & $9.695 \mathrm{E}-19$ & $1.306 \mathrm{E}+09$ & $3.817 \mathrm{E}-54$ & $3.420 \mathrm{E}+62$ & $9.204 \mathrm{E}+67$ & $3.716 \mathrm{E}-06$ \\
\hline $2.269 \mathrm{E}+30$ & $9.79 \mathrm{E}-29$ & $9.601 \mathrm{E}-20$ & $1.293 \mathrm{E}+08$ & $3.707 \mathrm{E}-57$ & $3.488 \mathrm{E}+64$ & $9.204 \mathrm{E}+69$ & $3.789 \mathrm{E}-06$ \\
\hline $2.269 \mathrm{E}+31$ & $9.99 \mathrm{E}-30$ & $9.483 \mathrm{E}-21$ & $1.277 \mathrm{E}+07$ & $3.572 \mathrm{E}-60$ & $3.575 \mathrm{E}+66$ & $9.204 \mathrm{E}+71$ & $3.884 \mathrm{E}-06$ \\
\hline $2.269 \mathrm{E}+32$ & $1.02 \mathrm{E}-30$ & $9.339 \mathrm{E}-22$ & $1.258 \mathrm{E}+06$ & $3.412 \mathrm{E}-63$ & $3.686 \mathrm{E}+68$ & $9.204 \mathrm{E}+73$ & $4.005 \mathrm{E}-06$ \\
\hline $2.269 \mathrm{E}+33$ & $1.04 \mathrm{E}-31$ & $9.166 \mathrm{E}-23$ & $1.234 \mathrm{E}+05$ & $3.225 \mathrm{E}-66$ & $3.827 \mathrm{E}+70$ & $9.204 \mathrm{E}+75$ & $4.158 \mathrm{E}-06$ \\
\hline $2.269 \mathrm{E}+34$ & $1.06 \mathrm{E}-32$ & $8.960 \mathrm{E}-24$ & $1.207 \mathrm{E}+04$ & $3.013 \mathrm{E}-69$ & $4.004 \mathrm{E}+72$ & $9.204 \mathrm{E}+77$ & $4.351 \mathrm{E}-06$ \\
\hline $2.269 \mathrm{E}+35$ & $1.08 \mathrm{E}-33$ & $8.719 \mathrm{E}-25$ & $1.174 \mathrm{E}+03$ & $2.776 \mathrm{E}-72$ & $4.229 \mathrm{E}+74$ & $9.204 \mathrm{E}+79$ & $4.595 \mathrm{E}-06$ \\
\hline $2.269 \mathrm{E}+36$ & $1.10 \mathrm{E}-34$ & $8.435 \mathrm{E}-26$ & $1.136 \mathrm{E}+02$ & $2.514 \mathrm{E}-75$ & $4.518 \mathrm{E}+76$ & $9.204 \mathrm{E}+81$ & $4.909 \mathrm{E}-06$ \\
\hline $2.269 \mathrm{E}+37$ & $1.12 \mathrm{E}-35$ & $8.103 \mathrm{E}-27$ & $1.091 \mathrm{E}+01$ & $2.229 \mathrm{E}-78$ & $4.896 \mathrm{E}+78$ & $9.204 \mathrm{E}+83$ & $5.319 \mathrm{E}-06$ \\
\hline $2.269 \mathrm{E}+38$ & $1.14 \mathrm{E}-36$ & $7.713 \mathrm{E}-28$ & $1.039 \mathrm{E}+00$ & $1.922 \mathrm{E}-81$ & $5.404 \mathrm{E}+80$ & $9.204 \mathrm{E}+85$ & $5.871 \mathrm{E}-06$ \\
\hline $2.269 \mathrm{E}+39$ & $1.16 \mathrm{E}-37$ & $7.249 \mathrm{E}-29$ & $9.762 \mathrm{E}-02$ & $1.596 \mathrm{E}-84$ & $6.117 \mathrm{E}+82$ & $9.204 \mathrm{E}+87$ & $6.646 \mathrm{E}-06$ \\
\hline $2.269 \mathrm{E}+40$ & $1.18 \mathrm{E}-38$ & $6.689 \mathrm{E}-30$ & $9.008 \mathrm{E}-03$ & $1.254 \mathrm{E}-87$ & $7.185 \mathrm{E}+84$ & $9.204 \mathrm{E}+89$ & $7.806 \mathrm{E}-06$ \\
\hline $2.269 \mathrm{E}+41$ & $1.20 \mathrm{E}-39$ & $5.991 \mathrm{E}-31$ & $8.067 \mathrm{E}-04$ & $9.006 \mathrm{E}-91$ & $8.958 \mathrm{E}+86$ & $9.204 \mathrm{E}+91$ & $9.732 \mathrm{E}-06$ \\
\hline $2.269 \mathrm{E}+42$ & $1.22 \mathrm{E}-40$ & $5.059 \mathrm{E}-32$ & $6.813 \mathrm{E}-05$ & $5.424 \mathrm{E}-94$ & $1.256 \mathrm{E}+89$ & $9.204 \mathrm{E}+93$ & $1.365 \mathrm{E}-05$ \\
\hline
\end{tabular}

\section{Application-8: To estimate the current cosmic rotational kinetic energy}

From assumptions $3 \mathrm{a}, 3 \mathrm{~b}$ and $3 \mathrm{c}$, current cosmic rotational energy can be estimated in the following way.

From classical mechanics, rotational kinetic energy of any spherical body is given by,

$$
K_{\text {rot }} \cong \frac{1}{2} I \omega^{2}
$$

where, $I$ is the moment of inertia of the rotating body and $\omega$ is the angular velocity. Based on this relation, current cosmic rotational kinetic energy can be expressed with the following relation.

$$
\left(K_{\text {rot }}\right)_{0} \cong \frac{1}{2} I_{0} \omega_{0}^{2} \cong \frac{1}{2} I_{0} H_{0}^{2}
$$

As current 'mass density' is very small in magnitude, current observable universe can be considered as a thin spherical shell and hence its corresponding current moment of inertia can be expressed with the following relation.

$$
I_{0} \cong \frac{2}{3} M_{0} R_{0}^{2}
$$

From the above two relations, current cosmic rotational kinetic energy can be expressed with the following simple relation. 


$$
\left(K_{r o t}\right)_{0} \cong \frac{1}{3} M_{0} R_{0}^{2} \omega_{0}^{2} \cong \frac{1}{3} M_{0} R_{0}^{2} H_{0}^{2} \cong 1.4257 \times 10^{72} \mathrm{~J}
$$

Surprisingly it is noticed that, ratio of proposed current cosmic rotational energy density and currently believed dark energy density is very close to unity. It can be expressed in the following way.

$$
\left[\frac{\left(K_{\text {rot }}\right)_{0}}{(4 \pi / 3) R_{0}^{3}}\right] /\left[0.68 \times\left(\frac{3 H_{0}^{2} c^{2}}{8 \pi G}\right)\right] \cong 0.98
$$

where $0.68 \times\left(\frac{3 H_{0}^{2} c^{2}}{8 \pi G}\right)$ is the currently believed dark energy density [23].

With reference to critical energy density, current rotational kinetic energy density can be expressed with the following relation.

$$
\frac{\left(K_{\text {rot }}\right)_{0}}{(4 \pi / 3) R_{0}^{3}} \cong \frac{M_{0} \omega_{0}^{2}}{4 \pi R_{0}} \cong \frac{M_{0} H_{0}^{2}}{4 \pi R_{0}}
$$

With respect to cosmic mass,

$$
\frac{\left(K_{\text {rot }}\right)_{0}}{(4 \pi / 3) R_{0}^{3}} \cong \frac{\omega_{0}^{2}}{4 \pi R_{0}}\left(\frac{c^{2} R_{0}}{G}\right) \cong \frac{H_{0}^{2}}{4 \pi R_{0}}\left(\frac{c^{2} R_{0}}{G}\right) \cong \frac{H_{0}^{2} c^{2}}{4 \pi G}
$$

Surprisingly it is noticed that, ratio of current cosmic rotational kinetic energy density and critical energy density is equal to $\frac{2}{3} \cong 0.666667$. It can be expressed in the following way.

$$
\left[\frac{\left(K_{r o t}\right)_{0}}{(4 \pi / 3) R_{0}^{3}}\right] /\left[\frac{3 H_{0}^{2} c^{2}}{8 \pi G}\right] \cong\left[\frac{H_{0}^{2} c^{2}}{4 \pi G}\right] /\left[\frac{3 H_{0}^{2} c^{2}}{8 \pi G}\right] \cong \frac{2}{3}
$$

If one is willing to consider this coincidence as a 'heuristic coincidence', it is possible to say that, currently believed 'dark energy' is nothing but the current cosmic rotational kinetic energy. It is for further study.

\section{Application-9: To estimate the current 'dark matter' density}

Based on the currently believed 'flat' model concept and current observations, 'dark matter' density can be fitted in the following way.. 


$$
\begin{aligned}
& \left(\rho_{\text {d.matter }}\right)_{0} \cong\left\{1-\left[\frac{\left(K_{\text {rot }}\right)_{0}}{\left[(4 \pi / 3) R_{0}^{3}\right] c^{2}}+\frac{M_{0}}{(4 \pi / 3) R_{0}^{3}}\right]\right\}\left(\frac{3 H_{0}^{2}}{8 \pi G}\right) \\
& \cong\left\{1-\left[\frac{2}{3}+0.048185\right]\right\}\left(\frac{3 H_{0}^{2}}{8 \pi G}\right) \cong 0.28515\left(\frac{3 H_{0}^{2}}{8 \pi G}\right)
\end{aligned}
$$

Quantitatively this can be compared with the currently believed 'dark matter' density [14] and needs further study. Proceeding further, we noticed a very interesting relation in the following way.

$$
\left(\rho_{\text {mass }}\right)_{0} c^{2} \cong \frac{\left[\left(\rho_{\text {mass }}\right)_{0} c^{2}+\left(\rho_{\text {d.matter }}\right)_{0} c^{2}\right]^{2}\left[\left(\rho_{\text {rot }}\right)_{0} c^{2}\right]^{2}}{\left[\left(\rho_{\text {critical }}\right)_{0} c^{2}\right]^{3}}
$$

where, $\quad\left(\rho_{\text {rot }}\right)_{0} c^{2} \cong \frac{\left(K_{\text {rot }}\right)_{0}}{(4 \pi / 3) R_{0}^{3}} \cong\left(\frac{2}{3}\right)\left(\frac{3 H_{0}^{2} c^{2}}{8 \pi G}\right)$. We are working on understanding this very strange and very simple relation with respect to past cosmological evolution. Based on this relation, if $\quad\left(\rho_{\text {mass }}\right)_{0} \cong 0.48185\left(\frac{3 H_{0}^{2}}{8 \pi G}\right)$, obtained $\left(\rho_{\text {d.matter }}\right)_{0} \cong 0.2811\left(\frac{3 H_{0}^{2}}{8 \pi G}\right)$.

\section{Discussion on Polarization rotation effect - an observable effect of cosmic rotation}

Yuri N. Obukhov and V.A. Korotky deeply discussed about the foundations and observational aspects of cosmic rotation. Readers are strongly encouraged to see the valuable paper [30]. According to Yuri N. Obukhov: the cosmic rotation affects a polarization of radiation which propagates in the curved spacetime and this produces a new observable effect which has been reported in the literature by Birch $[35,36]$ and Nodland and Ralston $[37,38]$.

In the geometrical optics approximation, polarization is described by a space-like vector $f^{\mu}$

which is orthogonal to the wave vector, $f_{\mu} k^{\mu}=0$, and is parallelly transported along the light ray,

$$
k_{\mu} \nabla_{\mu} f^{v}=0
$$

The influence of the gravitational field of a compact rotating massive body on the polarization of light was investigated by many authors [79-87]. The analysis of relation (39) for the rotating cosmologies shows that the global cosmic rotation also forces a polarization vector to change its orientation during the propagation along a null geodesics $[34,57,87]$. It is 
clear that this conclusion has physical meaning only when one can define a frame at any point of the ray with respect to which polarization rotates.

It is well known that, gravitational field affects all the properties of an image of a source, such as shape, size and orientation [89-93]. Like the rotation of the polarization vector, the deformation and rotation of an image depend both on the local coordinates and on the choice of an observer's frame of reference. However, if one considers a combination of the two problems, then a truly observable effect arises which is coordinate and frame independent. Putting it in another way, one should calculate the influence of the cosmic rotation on the relative angle $\eta$ between the polarization vector and the direction of a major axis of an image [31]. The relative angle $\eta$ can be most conveniently defined within the framework of the Newman-Penrose spin coefficient formalism.

With a detailed analysis and with the help of Kristian-Sachs expansions, it is possible to show that,

$$
\eta \cong \omega_{0} r \cos \theta+O\left(Z^{2}\right)
$$

This result is in a good agreement with the observational data reported [35] on the dipole anisotropy of distribution of the difference between the position angles of elongation (the major axis) and polarization in a sample of $3 \mathrm{CR}$ radio sources. The estimate for the direction and the magnitude of the cosmic rotation, obtained in [31,32,33] from Birch's data, reads

$$
\begin{aligned}
\left(l^{\circ}=\right. & \left.295^{\circ} \pm 25^{\circ} \text { and } b^{\circ}=24^{\circ} \pm 20^{\circ}\right) \\
& \rightarrow \omega_{0} \cong(1.8 \pm 0.8) H_{0}
\end{aligned}
$$

Here, we would like to suggest that, based on this kind of analysis, cosmic rotational effects can be studied and the ratio $\left[\frac{\omega_{0}}{H_{0}}\right]$ can be estimated. With further research, existence of cosmic rotation can be confirmed.

\section{Discussion on Cosmic horizon problem}

The 'horizon problem' or 'homogeneity problem' is a problem with the standard cosmological model of the hot Big Bang which was identified in the late 1960s, primarily by Charles W. Misner [94]. It points out that different regions of the universe have not 'contacted' each other because of the great distances between them, but nevertheless they have the same temperature and other physical properties. If one is willing to consider the concept of 'matter causes the space-time to curve', 'horizon problem' can be understood with $50 \%$. Remaining 50\% can be understood with assumptions 3 a to 3 f.collectively. 
According to hot big bang model, during its evolution, as universe is expanding, thermal radiation temperature decreases and matter content increases. As matter content increases, based on Mach's principle, i.e. (with assumption 3b), at any stage of evolution, it is possible to have an increasing radius of curvature, $R_{t} \cong \frac{G M_{t}}{c^{2}}$. Clearly speaking, as there exists no matter outside of $R_{t} \cong \frac{G M_{t}}{c^{2}}$, there is no scope for 'causal disconnection' As long as the universe is expanding with increasing super luminal speeds, cosmic age, cosmic radius and hence cosmic mass increases causing space to curve and probability of 'causal disconnection' decreases. Thus, with assumptions $3 \mathrm{a}$ to $3 \mathrm{f}$, currently believed 'cosmic horizon problem' can be resolved.

\section{Discussion on modern Cosmic inflation}

Here it is worth to pressent the views of Steinhardt PJ on 'inflation' [20]. In 1990s he was one the best creators of 'modern inflation'. In 2011, he is the best opponent of 'modern inflation'. Readers are strongly encouraged to see the web site: http://physics.princeton.edu/ steinh/0411036.pdf In his opinion:

The first signs that a theory is failing are usually small discrepancies between observations and predictions. That is not the situation here: the data are in exquisite accord with the inflationary predictions set down in the early 1980s. Instead the case against inflation challenges the logical foundations of the theory. Does the theory really work as advertised? Are the predictions made in the early 1980s still the predictions of the inflationary model as we understand it today? There is an argument to be made that the answer to both questions is no.

The first dictum holds that inflation is inevitable. But if it is, there is an awkward corollary: bad inflation is more likely than good inflation. "Bad inflation" means a period of accelerated expansion whose outcome conflicts with what we observe. For example, the temperature variations might be too large. The difference between good and bad hinges on the precise shape of the potential energy curve, which is controlled by a numerical parameter that could, in principle, take on any value whatsoever. Only an extremely narrow range of values could produce the observed temperature variation. In a typical inflationary model, the value must be near $10^{-15}$ - that is, zero to 15 decimal places. A less fine-tuned choice, such as zero to only 12 or 10 or eight decimal places, would produce bad inflation: the same degree of accelerated expansion (or more) but with a large temperature variation that is inconsistent with observations.

We could ignore bad inflation if it were incompatible with life. In that case, even if such large temperature variations could arise in principle, we could never observe them. Reasoning of this kind is known as the anthropic principle. Yet it does not apply here. Larger temperature variations would result in more stars and galaxies - the universe would, if anything, be more habitable than it is now. 
Not only is bad inflation more likely than good inflation, but no inflation is more likely than either. University of Oxford physicist Roger Penrose first made this point in the 1980s. He applied thermodynamic principles, similar to those used to describe configurations of atoms and molecules in a gas, to count the possible starting configurations of the inflaton and gravitational fields. Some of these configurations lead to inflation and thence to a nearly uniform, flat distribution of matter and a geometrically flat shape. Other configurations lead to a uniform, flat universe directly - without inflation. Both sets of configurations are rare, so obtaining a flat universe is unlikely overall. Penrose's shocking conclusion, though, was that obtaining a flat universe without inflation is much more likely than with inflation-by a factor of 10 to the googol $\left(10^{100}\right)$ power!

Another approach reaching a similar conclusion extrapolates the history of the universe from its current conditions backward in time using the established physical laws. The extrapolation is not unique: given the average flat and smooth conditions today, many different sequences of events could have come before. In 2008 Gary W. Gibbons of the University of Cambridge and Neil G. Turok of the Perimeter Institute for Theoretical Physics in Ontario showed that an overwhelming number of extrapolations have insignificant amounts of inflation. This conclusion is consistent with Penrose's. Both seem counterintuitive because a flat and smooth universe is unlikely, and inflation is a powerful mechanism for obtaining the needed smoothing and flattening. Yet this advantage appears to be completely offset by the fact that the conditions for starting inflation are so improbable. When all factors are taken into account, the universe is more likely to have achieved its current conditions without inflation than with it.

Many physicists and astrophysicists find these theoretical arguments unconvincing compared with a more compelling one favoring inflation: namely, the agreement between the predictions formulated in the early 1980s and the magnificent cosmological observations available today. Matching experiments trumps any theoretical argument. But the strange twist to this story is that the predictions of the early 1980s were based on a naive understanding of how inflation actually works - a picture that has turned out to be dead wrong.

In light of these arguments, the oft-cited claim that cosmological data have verified the central predictions of inflationary theory is misleading, at best. What one can say is that data have confirmed predictions of the naive inflationary theory as we understood it before 1983, but this theory is not inflationary cosmology as understood today. The naive theory supposes that inflation leads to a predictable outcome governed by the laws of classical physics. The truth is that quantum physics rules inflation, and anything that can happen will happen. And if inflationary theory makes no firm predictions, what is its point? The underlying problem is that procrastination carries no penalty - to the contrary, it is positively rewarded. Rogue regions that delay ending inflation continue to grow at an accelerating pace, so they invariably take over. In an ideal situation, any rogue regions would expand more slowly-or, better still, shrink. The overwhelming bulk of the universe would consist of well-behaved regions that end the smoothing phase on time, and our observed universe would be comfortably normal. An alternative to inflationary cosmology that my colleagues and I have proposed, known as the cyclic theory, has just this property. According to this picture, the big 
bang is not the beginning of space and time [see "The Myth of the Beginning of Time," by Gabriele Veneziano; Scientific American, May 2004] but rather a "bounce" from a preceding phase of contraction to a new phase of expansion, accompanied by the creation of matter and radiation. The theory is cyclic because, after a trillion years, the expansion devolves into contraction and a new bounce to expansion again. The key point is that the smoothing of the universe occurs before the bang, during the period of contraction. Any procrastinating rogue regions continue to contract while well-behaved regions bounce on time and begin expanding, so the rogue regions remain comparatively small and negligible.

Smoothing during contraction has an observable consequence. During any smoothing phase, whether in inflationary theory or in the cyclic theory, quantum fluctuations generate small, propagating random distortions in spacetime, known as gravitational waves, that leave a distinctive imprint on the microwave background radiation. The amplitude of the waves is proportional to the energy density. Inflation would occur when the universe was extremely dense, whereas the equivalent process in the cyclic model would occur when the universe was practically empty, so the predicted imprints would be dramatically different. Of course, the cyclic theory is relatively new and may have its own problems, but it illustrates that there are conceivable alternatives that may not suffer the uncontrollable runaway of eternal inflation. Our preliminary work suggests the cyclic model avoids the other problems described earlier, too.

To be sure, I have presented the cases for and against inflation as two extremes without the opportunity for cross-examination or nuance. In a meeting held in January at the Princeton Center for Theoretical Science to discuss these issues, many leading theorists argued that the problems with inflation are mere teething pains and should not shake our confidence in the basic idea. Others (including me) contended that the problems cut to the core of the theory, and it needs a major fix or must be replaced.

In the end, the case will be decided by data. The forthcoming observations of the microwave background radiation will be telling. Experiments to search for a gravitational-wave imprint are already being conducted on mountaintops, in high-altitude balloons and onboard satellites, and results should emerge within the next two to three years. Detecting a gravitational-wave imprint would support inflation; failure to detect it would be a major setback. For inflation to make sense despite a null result, cosmologists would need to suppose that the inflaton field had a very peculiar potential with just the right shape to suppress gravitational waves, which seems contrived. Many researchers would gravitate to alternatives, like the cyclic universe theory, that naturally predict an unobservably small gravitational-wave signal. The outcome will be a critical moment in our quest to determine how the universe came to be the way it is and what will happen to it in the future.

\section{Discussion on Quantum cosmology, the Planck scale Hubble parameter and the cosmic temperature}

In modern physics literature, one can find many articles on 'quantum cosmology'. One best review article is by Martin Bozowald [1]. In his opinion, 
1) "Quantum cosmology is based on the idea that quantum physics should apply to anything in nature, including the whole universe. Quantum descriptions of all kinds of matter fields and their interactions are well known and can easily be combined into one theory - leaving aside the more complicated question of unification, which asks for a unique combination of all fields based on some fundamental principles or symmetries. Nevertheless, quantizing the whole universe is far from being straightforward because, according to general relativity, not just matter but also space and time are physical objects. They are subject to dynamical laws and have excitations (gravitational waves) that interact with each other and with matter. Quantum cosmology is therefore closely related to quantum gravity, the quantum theory of the gravitational force and space-time. Since quantum gravity remains unfinished, the theoretical basis of quantum cosmology is unclear. And to make things worse, there are several difficult conceptual problems to be overcome".

2) "We remain far from a proper understanding of quantum cosmology, especially when physics at the Planck scale is involved. At the same time, research on quantum cosmology has led to progress in our understanding of generally covariant quantum systems and often showed unexpected effects of quantum space-time".

These points clearly indicate the poor status of 'current quantum cosmology'. In this context, proposed Planck scale Hubble parameter $H_{p l} \cong \sqrt{c^{5} / G \hbar} \cong 1.8549215 \times 10^{43} \mathrm{sec}^{-1}$ can be recommended for in depth analysis at fundamental level. In addition, one can see the simplicity of relation (2) in understanding and fitting the current Hubble parameter and current cosmic temperature in the form of relation (8) that seems to have a deep quantum mechanical back ground.

\section{Discussion on the proposed assumptions and their consequences}

We would like to highlight the following points:

1) Modern cosmologists strongly believe that current universe is accelerating. But they are silent in quantifying the past and current cosmic expansion speeds. Based on assumptuion $3 \mathrm{~d}$, we tried our level best in quantifying the past and current cosmic expansion speeds.

2) Even though, modern cosmology is strongly believing in current cosmic acceleration, it is silent in quantifying the current cosmic acceleraton. Along with the assumed cosmic age, we assume the current and initial cosmic expansion speeds and thereby estimating the past and current cosmic radii. With reference to data presented in table-1, it is very clear that, current cosmic expansion speed is slowly increasing with increasing cosmic age. Based on this fact, we can have two possible observations as expressed in the following way.

a) With increasing cosmic age, current universe is expanding with a slowly 
increasing speed of $11.885 c$.

b) Magnitude of the future cosmic expansion speed depends on the magnitude of the future Hubble parameter.

c) By knowing the time to time future cosmic temperatures, from relation (2), corresponding future Hubble parameters can be estimated and thus, corresponding future cosmic expansion speeds can be estimated.

3) With reference to the proposed assumptions, current universe seems to constitute 267 Hubble spheres. According to Mihran Vardanyan et al [25], the curvature scale of the Universe is conservatively constrained to be $R_{c}>42 \mathrm{Gpc}(99 \%)$, corresponding to a lower limit to the number of Hubble spheres in the Universe NU $>251$ (99\%). This coincidence clearly indicates the workability of our proposed assumptions.

4) We consider continuous superluminal expansions and hence it is possible to understand the currently observed large scale cosmic homogeniety or isotropy. Important point to be noted is that, modern estimate of cosmic radius is strictly assumed to obey 'inflation' whereas our estimate of cosmic radius (that is twice of modern estiamte) is independent of 'inflation'. From table-1, it is clear that, at around $1.06 \times 10^{-32}$ seconds, cosmic expansion speed was around $4.64 \mathrm{c}$ and at around 0.41 seconds, cosmic expansion speed was around 9.76c. Based on these points, 'inflation' concept can be relinquished [20]. In addition, concepts associated with 'fine tuning' of 'beginning of inflation' can also be ignored.

5) As the observed universe is very large and observers cannot reach outer parts of the univese, one may be forced to believe in 'temperature isotropy'.In reality, as universe is continuously assumed to be expanding at increasing super luminal speeds, expecting 'temperature isotropy' may not be reasonable.

6) Since it is assumed that, universe is always expanding with increasing super luminal speeds, generally believed 'Lambda term ' can be ignored in our proposed model.

7) Without a radial in-flow of matter in all directions towards one specific point, one cannot expect a big crunch and without a big crunch, one cannot expect a big bang. Really if there was a 'big bang' in the past, with reference to formation of big bang as predicted by GTR and with reference to the cosmic rate of expansion that might had taken place simultaneously in all directions at a 'naturally selected rate' about the point of big bang - 'point' of big bang can be considered as the characteristic reference point of cosmic expansion in all directions. Thinking in this way, to some extent, point of big bang can be considered as a possible center of cosmic expansion.

8) It may be noted that, many cosmologists are working on 'cosmic rotation' [30-70].In this context, one can see the following important points quoted by eminent scientists:

A) According to Fani Dosopoulou et al [41]: "Current observations are consistent with small amounts of universal rotation, which means that, if the universe rotates, it does so very slowly. This is also in agreement with the inflationary scenario, where the exponential expansion is expected to essentially eliminate any traces of primordial vorticity. Nevertheless, most (if not all) astrophysical structures rotate, which raises the question whether their rotation is of cosmological origin, or a 
relatively recent addition due to local physical processes. Magnetic fields have long been known to act as sources of rotational distortions and the agent responsible for their generation is the field's tension. Consequently, one could argue that the origin of cosmic magnetism and that of universal rotation are closely (if not directly) related. Put another way, magnetized universes should also rotate."

B) According to L.M.Chechin [42]: "From these estimations it result that Universe angular velocity must be in the following range $10^{-21} \mathrm{sce}^{-1} \leq \omega_{0} \leq 10^{-17} \mathrm{sce}^{-1}$. Our previous estimate of the Universe rotation $10^{-19} \mathrm{sce}^{-1}$, as it seems, is in good correlation with specified interval. At the same time the peculiarity of quoted articles is the Universe rotation searching in the determination by a baryonic substrate. In contrary, i.e the Universe rotation determines by Newtonian's mechanics and is the consequence of vacuum presence. That is why the ratio of Universe angular velocity to the Hubble parameter is about unit, i.e. $(\omega / H)_{0} \sim 1$.

From this follows the correctness of Gamov's remark [96] that the unique reason for Universe expansion and its rotation must be. If take into account that vacuum creates all baryonic substance, it is the moving force of the total Universe evolution, hence."

C) According to C.Sivaram et al [44]: "The origin of rotation or spin of objects, from stars to galaxies, is still an unanswered question. Even though there are models which try to explain this, none of them can account for the initial impulse that gave rise to this spin. In this paper we present that a cosmological model that contains a term involving the primordial spin of the universe can explain how these objects acquired the property of spin. This model also gives a natural explanation for the quadratic scaling of angular momentum with mass. Again, from this model, the background torsion due to a universal spin density not only give rise to angular momenta for all structures but also provide a background "centrifugal term" acting as a repulsive gravity accelerating the universe, with spin density acting as effective cosmological constant."

D) According to Serkan Zorba [45,46]: "The universe is rigidly rotating with an anular frequency equal to the Hubble's constant, and with a centrifugal force that is linearly proportional to distance. It implies that dark energy is not the "vacuum energy," but rather the rotational energy of the universe. My model thus has significant implications for the cosmological principle and the standard model of cosmology. Our universe appears to possess mysterious dark energy and dark matter because it is part of a rotating space-time of a disk-shaped universe, and the 
centrifugal and Coriolis forces due to rotation are perceived by us as dark energy and dark matter."

E) According to Wlodzimierz Godlowski [47]: “The presented observational situation is that the galaxies, their pairs and compact groups have a non-vanishing angular momentum. In the structures of mass corresponding to groups of galaxies, this feature has not been found, while in the clusters and super clusters alignment of galaxy orientation has been actually found. Also we know that galaxies have net angular momentum due to the fact that we actually measure the rotation curves of galaxies. These facts lead to the conclusion that theories which connect galaxy angular momentum with its surrounding structure are at some extend favored by data. We show that in the light of scenarios of galaxy formations this result could be interpreted as an effect of tidal forces mechanism, but it is also consistent with Li's model, in which galaxies form in the rotating universe."

F) According to Michael J. Longo [48]:“A preference for spiral galaxies in one sector of the sky to be left-handed or right-handed spirals would indicate a parity violating asymmetry in the overall Universe and a preferred axis. This study uses 15158 spiral galaxies with redshifts $z<0.085$ from the Sloan Digital Sky Survey. An unbinned analysis for a dipole component that made no prior assumptions for the dipole axis gives a dipole asymmetry of $-0.0408 \pm 0.011$ with a probability of occurring by chance of $7.9 \times 10^{-4}$. A similar asymmetry is seen in the Southern Galaxy spin catalog of Iye and Sugai. The axis of the dipole asymmetry lies at approx. $(l, b)=\left(52.0^{\circ}, 68.5^{\circ}\right)$, roughly along that of our Galaxy and close to alignments observed in the WMAP cosmic microwave background distributions. The observed spin correlation extends out to separations $\sim 210 \mathrm{Mpc} / \mathrm{h}$, while spirals with separations $<20 \mathrm{Mpc} / \mathrm{h}$ have smaller spin correlations."

G) According to Korotky V.A. and Obukhov [49]:“We believe that the cosmic rotation is an important physical effect which should find its final place in cosmology. In this paper we outlined one of the possible theoretical frameworks which can underlie our understanding of this phenomenon."

9) From modern estimates, cosmic radius about earth is 46.5 billion light years and from our estimate, cosmic radius about the point of big bang is 90 billion light years and ratio of these two radii is very close to $1 / 2$. Estimated radii point of view, factor $1 / 2$ is not a big issue. As earth is far away from the observable cosmic boundary, even though, if current universe is really rotating with very small magnitude of angular velocity, one may not be able to observe the effects of cosmic rotation from and about earth. 
10) Considering all the proposed assumptions collectively, it is certainly possible to show that, ratio of currently believed 'dark enery density' and proposed 'rotational kinetic energy density' is equal to unity. This coincidence casts doubt on the existence of 'dark energy' at fundamanetal level and needs further study. It may be noted that, very recently, Naser Mostaghel, in his paper [59] clearly demonstrated an alternative way of describing the expansion of space without involving the controversial dark energy. According to Xiaochun Mei and Ping Yu [60]: The red shift of cosmology is considered as the Doppler's effect. However, the basic formula used to calculate the high red shift of Ia supernova in current cosmology is related to scalar factor $R(t)$ rather than

velocity factor $\dot{R}(t)$. There exists inconsistency which is not allowed in physics. It is proved that the current formula used to calculates the relation of red shift and distance of Ia supernova is wrong in cosmology. We should directly use the Doppler's formula to calculate the red shift of cosmology. By the method of numerical calculation, based on the Newtonian gravity and the Doppler's formula, it is proved that the red shift of Ia supernova can be explained well. The hypotheses of dark energy and the accelerating expansion of the universe are completely unnecessary. The problem of the universe age can be solved well.

11) Even though our proposed model is independent of galactic redshifts, galactic distances and galactic receding speeds, with proposed assumptions, outline of the currently believed evolving cosmic structure can be understood very easily. By measuring the actual galactic receding speeds, assumption $3 \mathrm{~d}$ can be investigated further.

12) In any model of cosmology, fundamental questions to be solved are: 1) Why do 'dark matter' and 'visible matter' have their measured values of $\sim 33 \%$ of critical energy? 2) Why do 'dark energy' has its measured values of $\sim 68 \%$ of critical energy? 3) How to estimate their past and future magnitudes? These are the puzzling questions raised by the Royal Swedish Academy of Sciences [22] in 2011. In the conclusion part, Royal Swedish Academy of Sciences say: "The study of distant supernovae constitutes a crucial contribution to cosmology. Together with galaxy clustering and the CMB anisotropy measurements, it allows precise determination of cosmological parameters. The observations present us with a challenge, however: What is the source of the dark energy that drives the accelerating expansion of the Universe? Or is our understanding of gravity as described by general relativity insufficient? Or was Einstein's "mistake" of introducing the cosmological constant one more stroke of his genius? Many new experimental efforts are underway to help shed light on these questions". In this context, in applications 5, 6 and 7, we tried our level best in answering these basic questions with assumption $3 \mathrm{~b}$ which is having a long history in General relativity and Cosmology..

13) Believing in the existence of 'dark matter', stability of galactic clusters, galactic rotation speeds, oscillating patterns observed in $\mathrm{CMB}$ power spectrum, glaring inconsistency observed in Bullet cluster and patterns observed in galactic structural evolution can be understood. Important point to be noted is that, by estimating the 
current cosmic mass density and rotational kinetic energy, current dark matter percentage can be estimated accurately. See the proposed relations (37) and (38) in section-3, application-9.

14) We are working on applying our assumptions to review and understand the (big bang) nucleosynthesis. Based on the new approach and obtained results, scope and workability of our assumptions can be scrutinized and validated.

\section{Conclusions}

By following the proposed assumptions, we tried our level best to estimate and co-relate Hubble parameter, temperature, age, expansion speed, radius, mass, mass density, rotational kinetic energy, dark matter density and galactic receding speeds of the current expanding universe. Key points to be noted are:

1) We follow an evolving universe with incrreasing super luminal expansion speeds and super luminal rotational speeds.

2) We successfully implemented Planck scale in current cosmological observations.

3) We perfectly connected current Hubble parameter and current cosmic temperature.

4) We successfully implemented Mach's principle in modern cosmological observations.

5) We estimated and fitted current cosmic age, radius and mass desnity.

6) We estimated the current cosmic rotational kinetic energy and fitted with the currently believed dark energy.

7) We fitted the currently believed dark matter density with current mass density, rotational kinetic energy density and critical energy density.

8) We estimated and fitted CMBR radition temeperature, redshift and age.

9) We proposed two methods of understanding cosmic scale factor.

10) We proposed a very simple method for understanding current cosmic expansion speed.With reference to current cosmic temperature and current Hubble parameter, we assume that current universe is expanding with 11.885 times the speed of light. By knowing the time to time future cosmic temperatures, future Hubble parameters can be estimated and corresponding future cosmic expansion speeds can be estimated.

Proceeding further, with the proposed set of assumptions,

1) Extrapolation to past and future is very easy.

2) A unified model of evolving quantum cosmology can be developed.

3) With further study and observations, 'actual' galactic receding speeds and observed galactic redshits can be studied in a unified approach.

Acknowledgements: One of the authors, Seshavatharam U.V.S, is indebted to professors K.V. Krishna Murthy, Chairman, Institute of Scientific Research in Vedas (I-SERVE), Hyderabad, India and 
Shri K.V.R.S. Murthy, former scientist IICT (CSIR), Govt. of India, Director, Research and Development, I-SERVE, for their valuable guidance and great support in developing this subject.

\section{References}

[1] Martin Bozowald. Quantum cosmology: a review. Rep. Prog. Phys. 78 (2015) 023901

[2] Hawking, Stephen W. Quantum cosmology. In Hawking, Stephen W.; Israel, Werner. 300 Years of Gravitation. Cambridge University Press. 631-651. (1987)

[3] Luis J. Quantum gravity and minimum length. International Journal of Modern Physics A, vol 10, issue 2, pp. 145 (1995)

[4] Steffen Gielen and Neil Turok. Perfect Quantum Cosmological Bounce. Phys. Rev. Lett. 117, 021301 (2016)

[5] C. Rovelli and E. Wilson-Ewing, Why are the effective equations of loop quantum cosmology so accurate? Phys. Rev. D 90, 023538 (2014)

[6] Seshavatharam, U.V.S. and Lakshminarayana, S. Primordial Hot Evolving Black Holes and the Evolved Primordial Cold Black Hole Universe. Frontiers of Astronomy, Astrophysics and Cosmology, 1, 16-23. (2015)

[7] Seshavatharam, U. V. S. \& Lakshminarayana, S. On the Possible Role of Continuous Light Speed Expansion in Black Hole \& Gravastar Cosmology. Prespacetime Journal, Volume 7, Issue 3, pp. 584-600 (2016)

[8] Seshavatharam, U.V.S, Tatum, E.T and Lakshminarayana, S. On the role of gravitational self energy density in spherical flat space quantum cosmology. Journal of Applied Physical Science International. Vol.: 4, Issue.: 4, 228-236 (2015)

[9] G 't Hooft, Dimensional Reduction in Quantum Gravity, Utrecht Preprint THU-93/26, gr-qc/9310006. http://arxiv.org/abs/gr-qc/9310026v2 (1993/2009)

[10] Leonard Susskind. The World as a Hologram. J.Math.Phys.36:6377-6396 (1995)

[11] G. J. Whitrow. The Mass of the Universe. Nature 158, 165-166 (1946)

[12] D.W.Sciama. On the Origin of Inertia. MNRAS 113, 34, (1953)

[13] Dicke, R.H.: Gravitation-an enigma, American Scientist47, p. 25-40, (1959)

[14] Dicke, R.H .New Research on Old Gravitation: Are the observed physical constants independent of the position, epoch, and velocity of the laboratory? Science 6; 129 (3349):621-624. (1959)

[15] C. Brans and R. H. Dicke. Mach's principle and a relativistic principle of gravitation. Phys. Rev. 124, 925 (1961)

[16] Arbab I. Arbab. Quantization of Gravitational System and its Cosmological Consequences. Gen.Rel.Grav.36:2465-2479 (2004)

[17] Arbab I. Arbab. Large scale quantization and the essence of the cosmological problems, Spacetime \& Substance V.2, 55, (2001). (arXiv:physics/0102057v2)

[18] Berman M.S. Som.M.M. Whitrow-Randall's Relation and Brans-Dicke Cosmology. General Relativity and Gravitation, Vol 22, No 6, (1990)

[19] Guth AH. Inflationary universe: A possible solution to the horizon and flatness problems. Phys. Rev.;D23:347.(1981).

[20] Steinhardt PJ. The inflation debate: Is the theory at heart of modern cosmology deeply flawed? Scientific American.;304(4):18-25. (2011) 
[21] I. Bars, S. H. Chen, P. J. Steinhardt and N. Turok, Antigravity and the big crunch/big bang transition, Phys. Lett. B 715, 278-281, (2012)

[22] The accelerating universe. Compiled by the Class for Physics of the Royal Swedish Academy of Sciences.(2011). www.nobelprize.org/nobel_prizes/physics/laureates/2011/advanced-physicsprize2011.pdf

[23] Planck Collaboration: Planck 2015 Results. XIII. Cosmological Parameters.

[24] Adam G. Riess et al. A 2.4\% Determination of the Local Value of the Hubble Constant. Astrophys.J. 826 no.1. (2016)

[25] Mihran Vardanyan et al. Applications of Bayesian model averaging to the curvature and size of the Universe. MNRAS Lett 413, 1, L91-L95 (2011).

[26] Seshavatharam, U. V. S. \& Lakshminarayana, S . Is dark energy - an alias of cosmic rotational kinetic energy? International Journal of advanced astronomy. 4(2), 90-94 (2016)

[27] Seshavatharam, U. V. S. \& Lakshminarayana, S., Superluminal Expansion \& Rotation in Redshift Independent Accelerating Quantum Cosmology. Prespacetime Journal, Volume 7, Issue 11, pp. 1511-1534, (2016).

[28] Jeppe Trost Nielsen, Alberto Guffanti and Subir Sarkar. Marginal evidence for cosmic acceleration from Type Ia supernovae. arXiv:1506.01354v2 [astro-ph.CO] 9 Jun 2015.

[29] Alberto Guffanti. Università degli Studi di Torino \& INFN Torino. XXVIIIth Rencontres de Blois - Particle Physics and Cosmology Blois, 29 May - 3 June, 2016.

https://indico.cern.ch/event/464174/contributions/2170613/attachments/1283443/1907758/05_guf fanti.pdf.

[30] Yuri N. Obukhov. On physical foundations and observational effects of cosmic rotation. arXiv:astro-ph/0008106v1 7 Aug 2000.

[31] V.A. Korotky and Yu.N. Obukhov, Polarization of radiation in a rotating universe, JETP 81 (6) (1995) 1031-1035

[32] Yu.N. Obukhov, Rotation in cosmology, Gen. Relat. Grav. 24 (1992) 121-128.

[33] V.A. Korotky and Yu.N. Obukhov, Kinematical analysis of cosmological models with rotation, Sov. Phys. JETP 72(1) (1991) 11-15

[34] Yu.N. Obukhov, Observations in rotating cosmologies, in: "Gauge Theories of Fundamental Interactions" (Proceedings of the XXXII Semester in Stefan Banach Int. Math.Center, Warsaw, Poland, 1988)/Eds. R.R, aczka and M.Pawlowski (World Scientific: Singapore, 1990) 341-366.

[35] Birch P. Is the universe rotating?, Nature 298, 451(1982)

[36] P. Birch, Is there evidence for universal rotation? Birch replies, Nature 301 (1982) 736.

[37] B. Nodland and J.P. Ralston, Indication of anisotropy in electromagnetic propagation over cosmological distances, Phys. Rev. Lett. 78 (1997) 3043-3046.

[38] B. Nodland and J.P. Ralston, Appropriate null hypothesis for cosmological birefringenceReply, Phys. Rev. Lett. 79 (1997) 1958

[39] L.M. Chechin. Rotation of the Universe at different cosmological epochs. Astron.Rep. 60 no.6, 535-541. (2016)

[40] Demidchenko V. V. and Demidchenko V. I. The rotating universe. Liberal Arts in Russia.. Vol. 5. No. 2. Pp. 131-160. (2016)

[41] Fani Dosopoulou and Christos G. Tsagas. Vorticity survival in magnetized Friedmann universes. Phys.Rev.D89:103519,(2014)

[42] Chechin L.M. On the Modern Status of the Universe Rotation Problem. Journal of Modern Physics, 4, 126-132. (2013)

[43] Marcelo Samuel Berman, Fernando de Mello Gomide. Local and Global Stability of the Universe. Journal of Modern Physics, 4, 7-9, (2013) 
[44] Sivaram C, KenathArun. Primordial Rotation of the Universe, Hydrodynamics, Vortices and Angular Momenta of Celestial Objects. The Open Astronomy Journal, 5, 7-11(2012)

[45] Serkan Zorba. A Modified FRW Metric to Explain the Cosmological Constant. Mod. Phys. Lett. A, 27, 1250106 (2012)

[46] Serkan Zorba. Dark energy and dark matter as inertial effects. arXiv:1210.3021

[47] Wlodzimierz Godlowski. Global and local effects of rotation: Observational aspects. International Journal of Modern Physics D 201643 (2011)

[48] Michael J. Longo, Detection of a Dipole in the Handedness of Spiral Galaxies with Redshifts z 0.04, Phys. Lett. B.699:224-229 (2011)

[49] Vladimir A. Korotky and Yuri N. Obukhov. On cosmic rotation. Gravity, Particles and Space-Time, eds. P. Pronin and G. Sardanashvily, pp. 421-439. (World Scientific: Singapore, 1996)

[50] Sidharth B.G. Is the Universe Rotating? Prespacetime Journal. Vol. 1, Issue 7, pp. 1168-1173. (2010)

[51] Dmitri Rabounski. On the Speed of Rotation of Isotropic Space: Insight into the Redshift Problem. The Abraham Zelmanov Journal, Vol. 2, 208-223. (2009)

[52] Su S-C, Chu M-C. Is the universe rotating? Astrophysical Journal, 703 354. (2009).

[53] Chapline G, et al. Tommy Gold Revisited: Why Does Not The Universe Rotate? AIP Conf.Proc.822:160-165, (2006). http://arxiv.org/abs/astro-ph/0509230.

[54] Wlodzimierz Godlowski and Marek Szyd lowsk. Dark energy and global rotation of the Universe. Gen.Rel.Grav. 35, 2171-2187(2003)

[55] Robert V Gentry. New Cosmic Center Universe Model Matches Eight of Big Bang's Major Predictions Without the F-L Paradigm. CERN preprint, EXT-2003-022, (2003).

[56] Li-Xin Li. Effect of the Global Rotation of the Universe on the Formation of Galaxies. Gen.Rel.Grav. 30, 497(1998)

[57] Rainer W. Kuhne. On the Cosmic Rotation Axis. Mod.Phys.Lett.A12:2473-2474 (1997)

[58] Panov V. F., Sbytov, Yu. G., Accounting for Birch's observed anisotropy of the universe: cosmological rotation? Sov. Phys. JETP 74, 411(1992)

[59] Mostaghel, N. A New Solution for the Friedmann Equations. International Journal of Astronomy and Astrophysics, 6, 122-134 (2016)

[60] Xiaochun Mei, Ping Yu. The Formula to Calculate the Red Shift-Distance Relation of Ia Supernova in Cosmology Has Essential Mistakes - The Calculation of Cosmological Red Shift Should Use the Doppler Formula Directly. International Journal of Astronomy and Astrophysics, 2012, 2, 183-193.

[61] G.Ruben. Cosmic rotation and the inertial system. Astrophysics and Space Science 177(1):465-470 (1991)

[62] ÖYVIND GRÖN \& HARALD H. SOLENG Decay of primordial cosmic rotation in inflationary cosmologies. Nature 328, 501 - 503 (1987)

[63] Barrow JD, Juszkiewicz R, Sonoda DH. Universal rotation - How large can it be? Mon. Not. R. Astron. Soc. 213: 917. (1985)

[64] Valdes F., Tyson J. A., Jarvis J. F. Alignment of faint galaxy images: Cosmological distortion and rotation. Ap. J. 271, 431 (1983)

[65] Novello M, Reboucas MJ. Rotating universe with successive causal and noncausal regions. Phys. Rev. D 19, 2850-2852 (1979)

[66] F. M. Gomide. Cosmic Rotation and Mach's Principle in Three Special Cosmological Models. Revista Brasileira de Física, Vol. 3, No. 1, (1973) 
[67] Hawking S.W. On the rotation of the universe. Mon. Not. Royal. Astr. Soc. 142, 129- 141.(1969).

[68] J.V. Narlikar. Newtonian Universes With Shear and Rotation. MNRAS 126 (2): 203-208. (1963)

[69] Kurt Godel. Rotating Universes in General Relativity Theory. Proceedings of the international Congress of Mathematicians in Cambridge, 1: 175-81, (1950).

[70] Gamov G., Rotating Universe? Nature 158, 549. (1946).

[71] Hawking, S.W. Particle Creation by Black Holes. Commun. Math. Phys., 43: 199-220. (1975).

[72] U.V.S. Seshavatharam, Physics of Rotating and Expanding Black Hole Universe, Progress in Physics, vol. 2, pp7-14, (2010).

[73] U. V. S. Seshavatharam .The Primordial Cosmic Black Hole and the Cosmic Axis of Evil. International Journal of Astronomy 1(2): 20-37, (2012)

[74] U.V.S. Seshavatharam, S. Lakshminarayana. Friedmann cosmology: Reconsideration and New Results. International Journal of Astronomy, Astrophysics and Space Science, 1(2):16-26. (2014).

[75] Tatum, E.T., Seshavatharam, U.V.S. and Lakshminarayana, S. The Basics of Flat Space Cosmology. International Journal of Astronomy and Astrophysics, 5, 116-124 (2015)

[76] Tatum ET, Seshavatharam U.V.S, Lakshminarayana S. Flat space cosmology as a mathematical model of quantum gravity or quantum cosmology. International journal of astronomy and astrophysics. 5, 133-140. (2015).

[77] Tatum, E.T., Seshavatharam, U.V.S. and Lakshminarayana, S. Thermal Radiation Redshift in Flat Space Cosmology. Journal of Applied Physical Science International, 4, 18-26. (2015)

[78] Eugene Terry Tatum, U.V.S. Seshavatharam, and S. Lakshminarayana, Flat Space Cosmology as an Alternative to Lcdm Cosmology. Frontiers of Astronomy, Astrophysics and Cosmology, vol. 1, no. 2 (2015)

[79] G.V. Skrotskii, The influence of gravitation on the propagation of light, Sov. Phys.Doklady 2 (1957) 226-229.

[80] A.M. Volkov, A.A. Izmest'ev, and G.V. Skrotskii, The propagation of electromagnetic waves in a Riemannian space, Sov. Phys. JETP 32 (1971) 686-689

[81] J. Plebanski, Electromagnetic waves in gravitational fields, Phys. Rev. 118 (1960)1396-1408.

[82] A.M. Anile and R.A. Breuer, Gravitational Stokes parameters, Astrophys. J. 189 (1974) 39-49.

[83] A.M. Anile and S. Motta, Perturbations of the general Robertson-Walker universes and angular variations of the cosmic blackbody radiation, Astrophys. J. 207 (1976) 685-692.

[84] F. Fayos and J. Llosa, Gravitational effects on the polarization plane, Gen. Rel. Grav.14 (1982) 965-978

[85] N.Yu. Gnedin and I.G. Dymnikova, Rotation of the plane of polarization of a photon in a Petrov type D space-time, Sov. Phys. JETP 67 (1988) 13-15

[86] I.Yu. Kobzarev and K.G. Selivanov, Rotation of the optical polarization vector in a nonstationary gravitational field, Sov. Phys. JETP 67

[87] B. Mashhoon, Influence of gravitation on the propagation of electromagnetic radiation, Phys. Rev. D11 (1975) 2679-2684

[88] R. Mansouri and K. Nouzari, Rotation of plane of polarization of an electromagnetic wave propagating over cosmological distances in "Finite rotating universe" scenario, Preprint Institute for Studies in Physics and Mathematics, Tehran, Iran (1997) 4 p.;e-print Archive: astro-ph/9710028.

[89] R. Sachs, Gravitational waves in general relativity. IV. The outgoing radiation condition, Proc. Roy. Soc. A264 (1961) 309-338.

[90] I.D. Novikov, V.P. Frolov, Physics of black holes (Nauka: Moscow, 1986), in Russian 
[91] G.F.R. Ellis, Relativistic cosmology, in: General Relativity and Cosmology (Rendiconti S.I.F., Corso XLVIII, Varenna, 1969), Ed. R.K. Sachs (Academic Press: New York, 1971) 104-182

[92] G.F.R.Ellis, Relativistic cosmology, in: Carg 'ese Lect. in Phys. vol. 6, Ed. E. Schatzman (Gordon and Breach: NY, 1973) 1-60.

[93] S. Seitz, P. Schneider, and J. Ehlers, Light propagation in arbitrary spacetimes and the gravitational lens approximation, Class. Quantum Grav. 11 (1994) 2345-2373.

[94] Charles W. Misner, Kip S. Thorne. John Archibald Wheeler, Gravitation. W.H. Freeman and Company, (1973)

(C) 2016 by the authors; licensee Preprints, Basel, Switzerland. This article is an open access article distributed under the terms and conditions of the Creative Commons by Attribution (CC-BY) license (http://creativecommons.org/licenses/by/4.0/). 